\title{
ESTRUCTURA, COMPOSICIÓN Y DIVERSIDAD DE LA SELVA baja Caducifolia del Cerro Verde, Nizanda (OAXACA), MÉXICO
}

\author{
José A. Gallardo-Cruz', Jorge A. Meave y Eduardo A. Pérez-García \\ Departamento de Ecología y Recursos Naturales, Facultad de Ciencias, Universidad Nacional Autónoma de México, \\ Circuito Exterior s/n, Ciudad Universitaria, México 04510, D.F., México. \\ 1 Autor para la correspondencia. Tel 5622-4835; correo-e: jagc@ciencias.unam.mx
}

\begin{abstract}
Resumen: En este estudio se describe la estructura, la composición florística y los patrones de diversidad de la selva baja caducifolia del Cerro Verde, localizado en Nizanda (Istmo de Tehuantepec, Oaxaca, México). En 30 parcelas de $100 \mathrm{~m}^{2}$ se censaron las plantas leñosas con DAP $\geq 1 \mathrm{~cm}$ (estrato alto), y en cinco subcuadros de $4 \mathrm{~m}^{2}$ dentro de cada una se censaron las plantas con DAP $<1 \mathrm{~cm}$, pero con altura $>30 \mathrm{~cm}$ (estrato bajo). Se encontraron 194 especies distribuidas en 52 familias. La riqueza de especies fue idéntica en ambos estratos (145 especies), y cada uno aportó de manera exclusiva ca. 25\% de la riqueza total registrada. Leguminosae fue la familia más rica en especies (27), seguida por Asteraceae (20) y Euphorbiaceae (18). Las especies más frecuentes fueron Bursera simaruba $+B$. aff. cinerea (no distinguidas en el campo), Euphorbia schlechtendalii, Pilosocereus collinsii y Capparis verrucosa. La riqueza específica promedio por cuadro fue de 30 especies. Los índices basados en la riqueza y la abundancia de las especies (Berger-Parker $=0.20 ;$ Simpson $=0.09 ; \alpha$ de Fisher $=9.47 ;$ Shannon $=2.78 ;$ equitatividad $=0.82$ ) indican una alta diversidad y una consecuente baja dominancia en esta selva. Los valores extrapolados mostraron una densidad total (estratos alto + bajo) de 23,950 ind. ha ${ }^{-1}\left(830\right.$ ind. ha ${ }^{-1}$ con DAP $\geq 10 \mathrm{~cm}$ ), una cobertura de $446.7 \%$, y un área basal de 53 $\mathrm{m}^{2} \mathrm{ha}^{-1}$. La altura promedio del $10 \%$ de los árboles más altos fue $9.1 \mathrm{~m}$. La estructura de la selva baja del Cerro Verde es semejante a la de otras comunidades vegetales del trópico seco de México y denota un buen estado de conservación. Este hecho, aunado a su particular composición florística, enfatiza la pertinencia de su conservación formal.

Palabras clave: área basal, análisis estructural, bosque tropical caducifolio, cobertura, densidad, florística, Istmo de Tehuantepec, vegetación tropical.
\end{abstract}

\begin{abstract}
In this study we describe the structure, floristic composition, and diversity of the tropical dry forest established on the Cerro Verde (Verde Hill), located at Nizanda (Isthmus of Tehuantepec, Oaxaca, Mexico). We censused woody plants with DBH $\geq 1 \mathrm{~cm}$ (upper stratum) present in $30100-\mathrm{m}^{2}$ plots, and in five 4- $\mathrm{m}^{2}$ subplots within each of them, those plants with height $>30$ $\mathrm{cm}$ but $<1 \mathrm{~cm} \mathrm{DBH}$ (lower stratum). A total of 194 species belonging to 52 families were recorded. Species richness was identical in both strata (145 species), each contributing exclusively with $c a$. $25 \%$ of total recorded richness. Leguminosae was the most speciose family (27), followed by Asteraceae (20) and Euphorbiaceae (18). The most frequent species were Bursera simaruba $+B$. aff. cinerea (not distinguished in the field), Euphorbia schlechtendalii, Pilosocereus collinsii and Capparis verru$\cos a$. Average richness by plot was 30 species. Mean values of indices based on species richness and abundances (Berger-Parker $=0.20$; Simpson $=0.09$; Fisher's $\alpha=9.47$; Shannon $=2.78$; eveness $=0.82$ ) indicated a high diversity and consequently a low dominance in this forest. Extrapolated values showed a total (upper + lower strata) density of 23,950 ind. ha ${ }^{-1}\left(830 \mathrm{ind}\right.$. ha ${ }^{-1}$ with $\mathrm{DBH} \geq 10 \mathrm{~cm}$ ), a forest cover of $446.7 \%$, and a basal area of $53 \mathrm{~m}^{2} \mathrm{ha}^{-1}$. Mean height of those individuals accounting for $10 \%$ of the highest trees by plot was $9.1 \mathrm{~m}$. Tropical dry forest structure at Cerro Verde is comparable to other Mexican communities within the seasonally dry tropical region and indicates a good conservation status. This feature, together with its particular floristic composition, makes of this area a high priority site which requires inclusion in a formal conservation scheme.
\end{abstract}

Key words: basal area, density, floristics, forest cover, Isthmus of Tehuantepec, structural analysis, tropical deciduous forest, tropical vegetation.

$\mathbf{E}$ 1 estudio de la vegetación tropical de México muestra un sesgo notable hacia las regiones más húmedas de su vertiente atlántica. Por lo tanto, la información disponible para la vegetación de las regiones estacionalmente secas, localizadas principalmente a lo largo del litoral pacífico del sur y oeste de México, todavía es pobre. Esta situación es paradójica si se considera que la porción tropical seca del país ocupa un área considerablemente mayor que la húmeda (Rzedowski, 1978; Challenger, 1998; Trejo y Dirzo, 2000; Palacio-Prieto et al., 2000). 
Además de escaso, el conocimiento cuantitativo sobre la vegetación tropical seca es fragmentado, ya que proviene de unas cuantas localidades o regiones relativamente bien conocidas pero distantes entre sí. Entre ellas destaca la región de Chamela, cuya vegetación es sin duda la más estudiada del país en su tipo (e.g. Lott et al., 1987; Martínez-Yrízar et al., 1992, 1996; Balvanera et al., 2002; Durán et al., 2002). Después de los estudios pioneros de Faustino Miranda $(1942,1947,1952)$ en diversas regiones tropicales del país y de Harold Scott Gentry (1942) para la cuenca del río Mayo, no fue sino hasta finales del siglo XX que se comenzó a acumular una cantidad significativa de conocimiento para otras regiones, por ejemplo el sur de Sonora (Búrquez et al., 1999; Martin et al., 2000; Martínez-Yrízar et al., 2000), la costa de Oaxaca (SalasMorales, 2002) y la Península de Yucatán (Rico-Gray et al.,
1988; White y Hood, 2004). El único estudio que ha intentado generar información cuantitativa comparable para un gran número de localidades del trópico seco mexicano es el de Trejo y Dirzo (2002). No obstante estos esfuerzos, llama la atención la casi total carencia de datos para la región del Istmo de Tehuantepec, en la porción oriental del estado de Oaxaca.

La falta de información para la región istmeña es lamentable, ya que ésta ha sido reconocida por su gran importancia biológica (Acosta-Castellanos, 1995; Pérez-García et al., 2001), sobre todo por su alto nivel de endemismo (Lorence y García-Mendoza, 1989; Torres-Colín, 2004), y por ser uno de los pocos puntos del continente donde han tenido contacto las biotas propias de las vertientes atlántica y pacífica (Rzedowski, 1962). Consecuentemente, es posible que las comunidades bióticas allí asentadas reflejen en

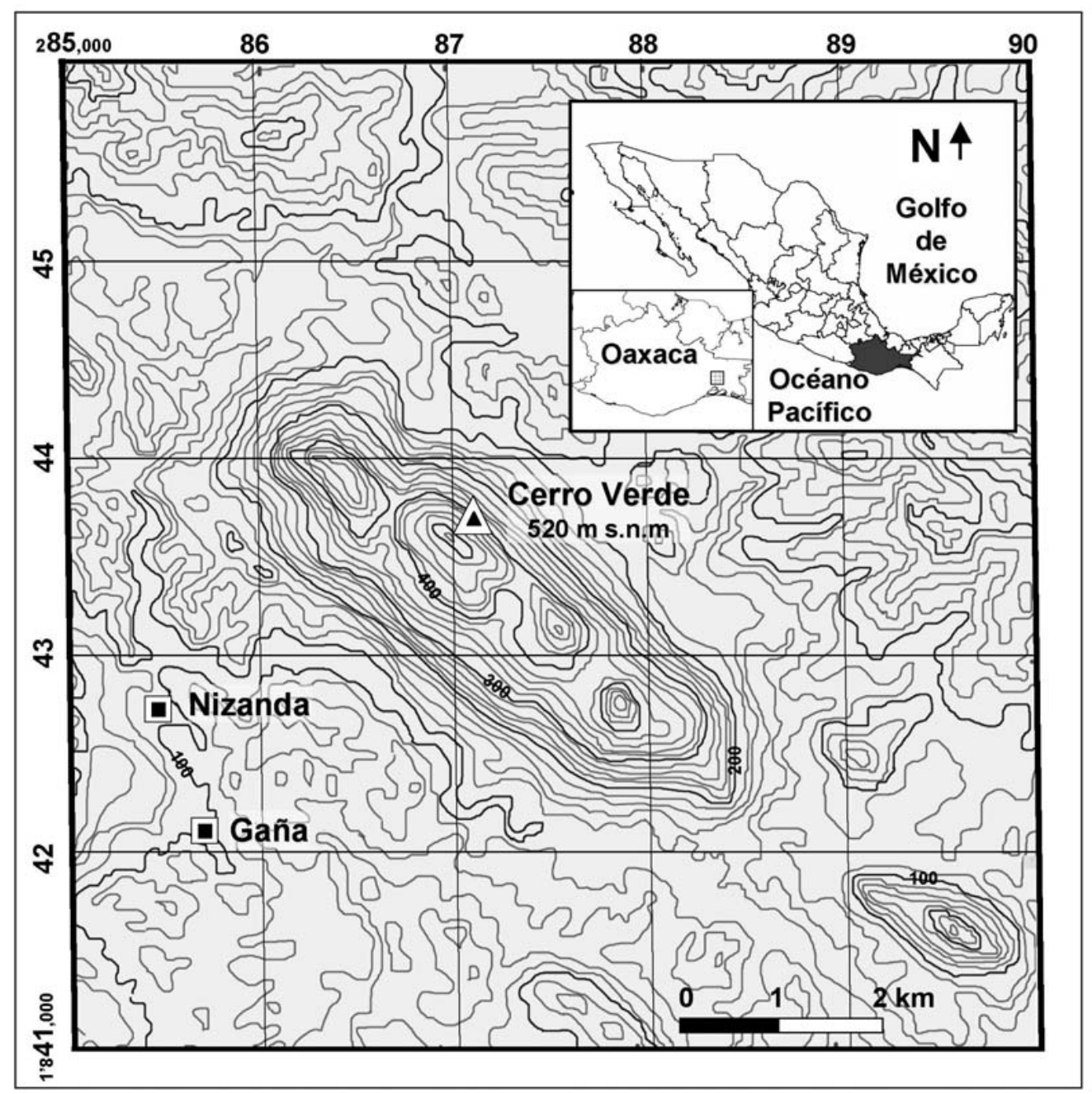

Figura 1. Localización y curvas de nivel del Cerro Verde. Las coordenadas UTM corresponden a la Zona 15. 


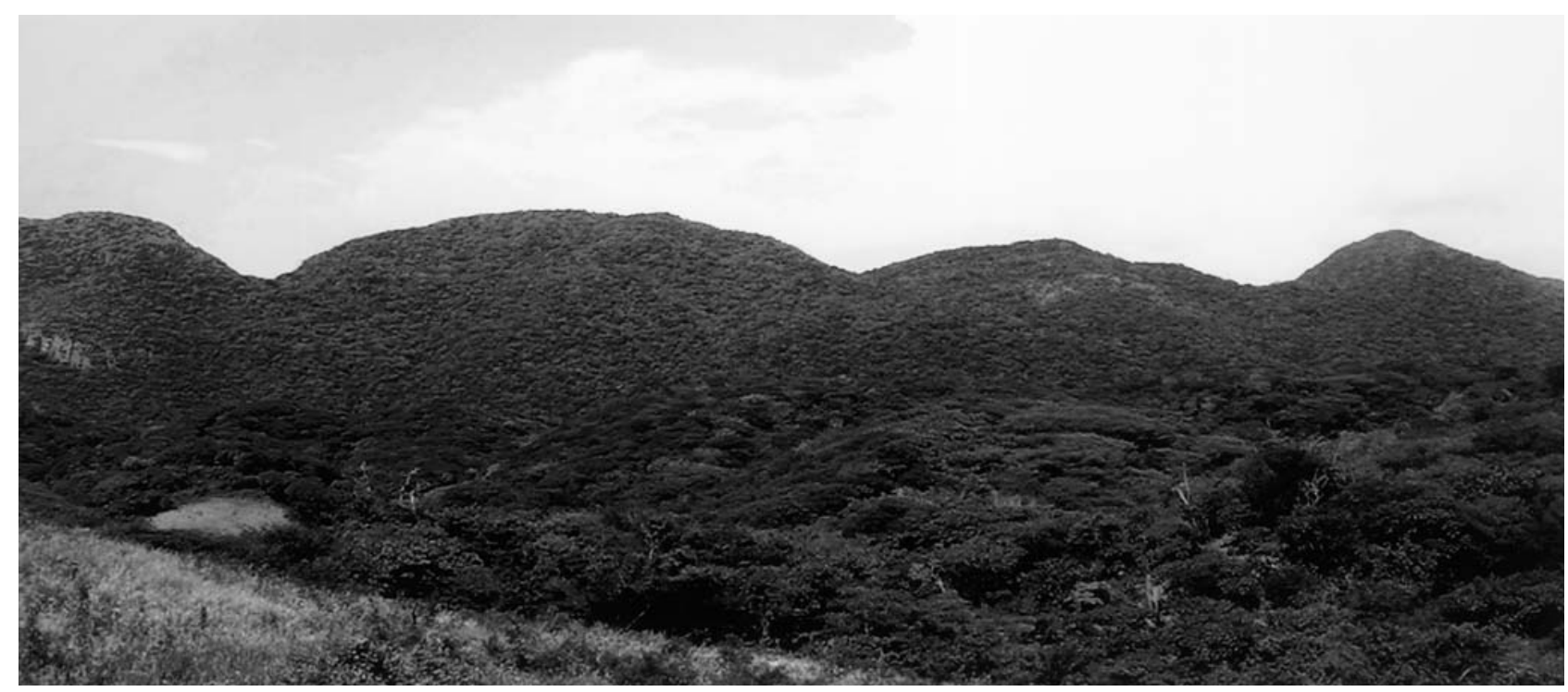

Figura 2. Vista del Cerro Verde desde su lado sur, mostrando las crestas que lo caracterizan.

cierta medida esta situación y no se asemejen a otras comunidades análogas del país. Por otro lado, las comunidades vegetales del Istmo están en una posición relativamente marginal respecto a las grandes áreas de selva baja caducifolia (SBC), sensu Miranda y Hernández-X. (1963) que existen en México, por lo que es posible que la flora que las compone difiera sustancialmente respecto a las comunidades similares de otras regiones.

Estas razones dificultan hacer predicciones sobre los patrones de diversidad de las comunidades de SBC de esta región. Por ejemplo, es posible que las relaciones de dominancia al interior de las comunidades sean diferentes, o que el contingente de especies raras o subordinadas no esté igualmente representado que en otras regiones. Asimismo, cabe esperar que estas peculiaridades se manifiesten no sólo a nivel de especie, sino que también se expresen en los patrones de diversidad correspondientes a taxa superiores.

El presente estudio ofrece una descripción de la estructura, la composición florística y los patrones de diversidad de la selva baja caducifolia que se desarrolla en el Cerro Verde, elevación que a pesar de su altitud moderada, destaca en las tierras bajas que predominan en el Istmo de Tehuantepec. Con esta información se hizo una comparación de las características de la SBC de este sitio con otras localidades mexicanas, con el objeto de comenzar a identificar patrones en este tipo de vegetación, y se hizo una valoración de la pertinencia de su conservación.

\section{Área de estudio}

El Cerro Verde (16³9'48.1” N y 9459'50.8” O) se localiza en los terrenos del ejido de Nizanda, ubicado en la porción sur del Istmo de Tehuantepec (Municipio de Asunción
Ixtaltepec, Distrito de Juchitán, Oaxaca), sur de México (figuras 1,2).

El relieve típico de la región se caracteriza por presentar lomeríos de filitas de baja elevación ( $\approx 250 \mathrm{~m} \mathrm{snm}$ ), aunque existen afloramientos calcáreos que irrumpen en el paisaje. El Cerro Verde, que es el afloramiento más prominente de este tipo, cubre un área de $3.5 \mathrm{~km}^{2}(3.2 \times 1.1 \mathrm{~km}$ en sus ejes más largo y ancho, respectivamente). Presenta cuatro crestas en el eje mayor, cuyas altitudes fluctúan entre 460 y $500 \mathrm{~m}$. Debido a su forma oblonga y a la orientación de su eje principal (NOO - SEE), el Cerro Verde tiene dos caras principales, una expuesta al noreste $\left(38^{\circ}\right)$ y otra al suroeste $\left(218^{\circ}\right)$. Entre ellas se observa una cierta asimetría: la orientada al SO tiene una pendiente más pronunciada y su límite altitudinal inferior se encuentra a $100 \mathrm{~m}$, mientras que la ladera NE tiene una pendiente más suave y su base está a 120 m snm (Gallardo-Cruz, 2004).

A partir de información de estaciones meteorológicas cercanas a Nizanda, Pérez-García et al. (2001) estimaron una temperatura media anual de $25^{\circ} \mathrm{C}$ y una precipitación total anual promedio de aprox. 1,000 $\mathrm{mm}$; el tipo climático probable de la región es Awo(w)igw", es decir, un clima cálido subhúmedo con lluvias en verano (García, 1988). La estacionalidad en la precipitación se refleja en la existencia de una época seca que se extiende de noviembre a mayo, y una húmeda, con presencia de canícula, que abarca de junio a octubre (Anónimo, 1984a, b). Un factor particularmente importante en la determinación del clima regional es la presencia de corrientes eólicas de gran velocidad, debidas al paso forzado de los vientos alisios entre las serranías que circundan las partes bajas del Istmo (Romero-Centeno et al., 2003).

La litología superficial está constituida por calizas y fili- 
tas de edad mesozoica, y los tipos de suelos presentes son Litosoles, Feozems háplicos y Regosoles éutricos (Anónimo, 1981). En el Cerro Verde existe un mosaico edáfico definido por la alternancia de sitios con suelo de profundidad variable y manchones de roca caliza expuesta. En la parte baja de las laderas los suelos son arcillosos, relativamente profundos $(>50 \mathrm{~cm})$, y presentan tonalidades rojizas en los horizontes inferiores. En las zonas de altitud intermedia, los suelos se hacen más someros $(<50$ $\mathrm{cm}$ ) y presentan un horizonte A bien desarrollado, cubierto por una gruesa capa de mantillo, y un horizonte B poco diferenciado.

En las porciones donde el suelo está mejor desarrollado se establece la SBC, que es el tipo de vegetación predominante en el Cerro Verde. Por encima de la cota de $430 \mathrm{~m}$ el sustrato consiste básicamente en roca expuesta, y allí la vegetación está conformada por matorral xerófilo (MX) y selva baja caducifolia en roca (SBCr), descritos por PérezGarcía y Meave (2004). Por ser poco propicio para las actividades agropecuarias, en el Cerro Verde se encuentra uno de los remanentes de vegetación mejor conservados de esta región.

\section{Materiales y métodos}

El trabajo de campo se efectuó entre julio y septiembre de 2001. Los datos de la vegetación fueron registrados en 30 cuadros de $10 \times 10 \mathrm{~m}\left(100 \mathrm{~m}^{2}\right)$, distribuidos de manera equitativa en las dos laderas principales del cerro. Durante la selección de los sitios de muestreo se evitaron lugares con indicios de perturbación o donde la roca caliza estuviera expuesta.

Para el muestreo de la vegetación se distinguieron $a$ priori dos estratos: uno alto formado por todas las plantas leñosas o herbáceas que tuvieran un diámetro a la altura del pecho (DAP; $1.3 \mathrm{~m}$ ) $\geq 1 \mathrm{~cm}$, y un estrato bajo conformado por plantas que no alcanzaban este diámetro pero que tuvieran una altura $>30 \mathrm{~cm}$. En ambos casos, se anotó la identidad taxonómica y se midieron (al $\mathrm{cm}$ más cercano) dos diámetros de cobertura y la altura total de las plantas enraizadas en su respectivo cuadro. Además, a las plantas del estrato alto se les midió el DAP y se anotó si eran policaulescentes. Las plantas del estrato bajo fueron muestreadas en cinco subcuadros de $4 \mathrm{~m}^{2}\left(2 \times 2 \mathrm{~m} ; 20 \mathrm{~m}^{2}\right.$ en total) localizados en las esquinas y en el centro del cuadro de $100 \mathrm{~m}^{2}$. Para el análisis estructural del estrato bajo, los valores de densidad y cobertura fueron extrapolados a un área de $100 \mathrm{~m}^{2}$. Debido al difícil acceso a los cuadros de muestreo por lo abrupto del terreno, el tiempo para el muestreo estuvo limitado, lo que obligó a excluir a las plantas epífitas del estudio.

La determinación taxonómica se realizó por medio del cotejo de los ejemplares recolectados con los depositados en una colección de referencia de la región de Nizanda, res- guardada en el Departamento de Ecología y Recursos Naturales (Facultad de Ciencias, UNAM). Este procedimiento permitió elaborar una lista de las especies presentes en todos los cuadros, siguiendo los sistemas de clasificación a nivel de familia de Magnoliopsida (Cronquist, 1981) y Liliopsida (Dahlgren et al., 1985), y la nomenclatura de géneros y especies empleada por Pérez-García et al. (2001). La única excepción a estos criterios fue el reconocimiento de la familia Leguminosae (sensu lato), en donde se agruparon a las tres familias reconocidas por Cronquist, debido a la dificultad de distinguirlas en los ejemplares estériles.

El análisis estructural incluyó el cálculo de densidades, coberturas y áreas basales, utilizando procedimientos convencionales (Mueller-Dombois y Ellenberg, 1974; Matteucci y Colma, 1982); para las dos últimas variables, los cálculos se hicieron por individuo, especie y cuadro. Para el análisis de la altura se definieron dos conjuntos de individuos en cada cuadro, uno comprendido por todos los individuos del estrato alto y otro formado por el $10 \%$ de los árboles más altos de este mismo estrato (Salas-Morales, 2002). Para ambos conjuntos se calcularon las alturas promedio, a las cuales se les denominó "altura media" y "altura $10 \%$ superior", respectivamente.

El análisis de la diversidad por cuadro y en total para la comunidad se basó en primer lugar en la riqueza de especies (S). Además, se calcularon los índices de BergerParker (d) y de Simpson (D) como medidas de dominancia (Magurran, 1988), y como medidas de diversidad ecológica el índice de Shannon (H') y su equitatividad (E), y el índice $\alpha$ de Fisher (Hayek y Buzas, 1997).

\section{Resultados}

Composición florística. La lista florística del Cerro Verde incluye 194 morfoespecies (referidas a partir de aquí como especies) que representan a 52 familias de la división Magnoliophyta. De ellas, 141 (72.3\%) fueron determinadas hasta el nivel de especie y $16(8.2 \%)$ hasta el nivel de género. De las especies restantes, $34(17.9 \%)$ sólo se pudieron determinar a nivel de familia y tres $(1.5 \%)$ permanecieron como desconocidas. La riqueza de especies fue exactamente igual en cada uno de los dos estratos, ya que en ambos aparecieron 145 especies (apéndice 1). Las especies que aparecieron únicamente en el estrato bajo se enlistan en el apéndice 2. A pesar de las especies compartidas, cada estrato aportó de forma exclusiva aproximadamente $25 \%$ de la riqueza total registrada para el sistema.

La distribución de las especies por familia fue muy desigual (figura 3). Las 13 familias (25\%) más ricas (i.e. con cinco especies o más) agruparon en conjunto 124 especies (63.5\% de la riqueza total). Por el contrario, las 22 familias $(42.3 \%)$ representadas por una sola especie sólo contribuyeron con $11.2 \%$ de la riqueza total. Con 27 


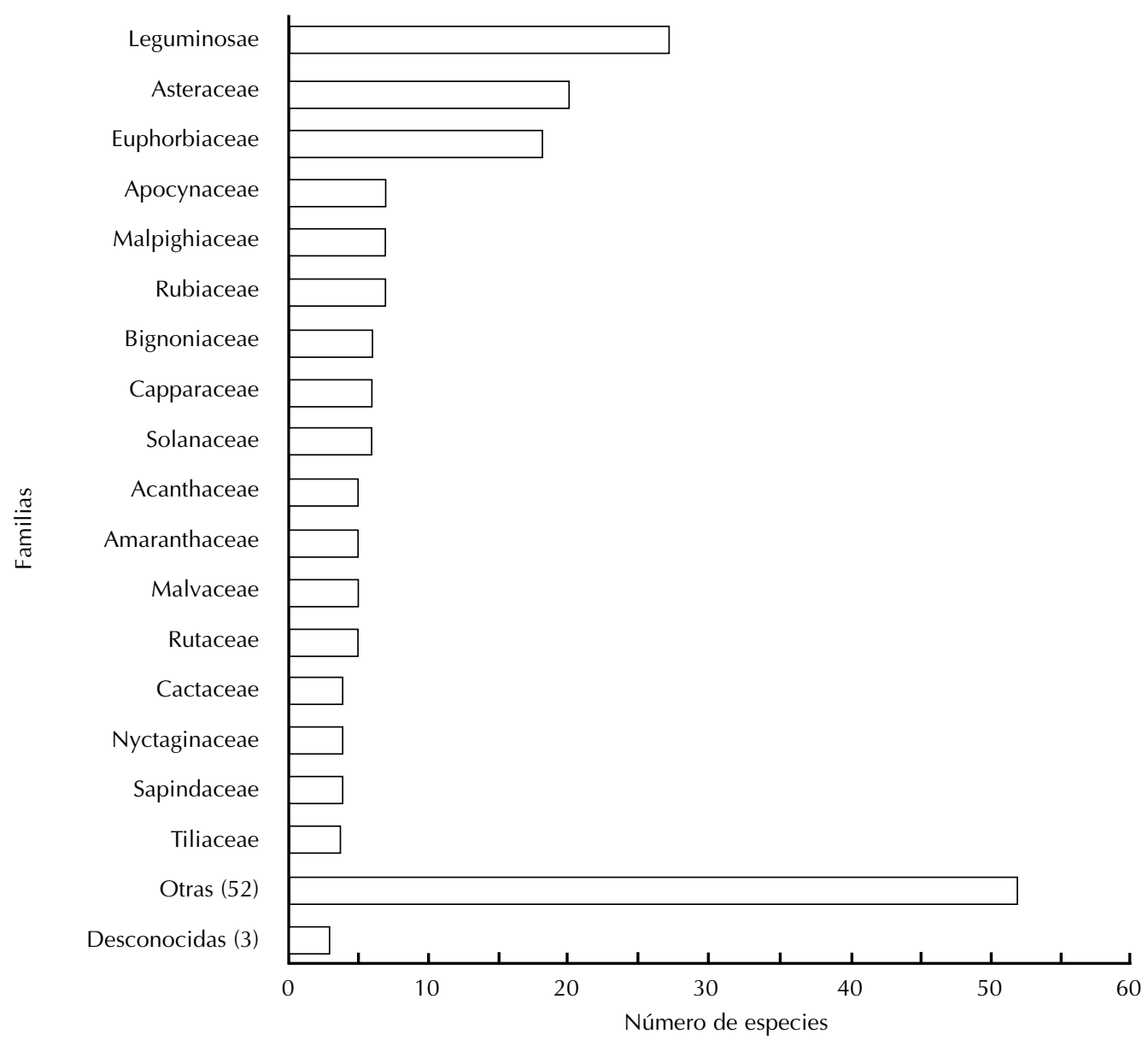

Figura 3. Distribución de especies de las familias más diversas registradas en 30 cuadros de muestreo ( 0.3 ha) de SBC en el Cerro Verde, región de Nizanda, Oaxaca.

especies, Leguminosae fue la familia con mayor riqueza específica; a ésta le siguieron Asteraceae (20 especies), Euphorbiaceae (18), y Apocynaceae, Malpighiaceae y Rubiaceae (7 cada una).

Las familias con mayor abundancia de individuos $(\geq$ 300) fueron Euphorbiaceae (1,476 ind.), Acanthaceae (1,103), Leguminosae (1,076), Malvaceae (474) y Asteraceae (331). Estas cinco familias incluyeron en conjunto $62 \%$ de todos los individuos registrados en el estudio. Otras familias importantes por sus abundancias fueron Malpighiaceae (280 ind.), Amaranthaceae (265), Rutaceae (238), Cactaceae (160) y Burseraceae (148).

Ninguna especie apareció en todos los cuadros de muestreo. Las especies con las frecuencias más altas fueron Bursera simaruba + B. aff. cinerea (Burseraceae; consideradas como una sola entidad ya que no pudieron ser distinguidas en el campo), Euphorbia schlechtendalii (Euphorbiaceae), Pilosocereus collinsii (Cactaceae) y Capparis verrucosa (Capparaceae), registradas en 26, 25, 21 y 20 cuadros, respectivamente. Casi tres cuartas partes
(71.8\%) de las especies aparecieron en cinco cuadros de muestreo o menos, y casi la mitad de éstas (34.3\%) fueron encontradas sólo en uno.

Diversidad florística. La riqueza específica promedio $( \pm 1$ E.E.) por cuadro fue $30 \pm 0.90$ especies (intervalo $=21-39$ especies). Los promedios de los índices de Berger-Parker $(0.20 \pm 0.01)$ y de Simpson $(0.09 \pm 0.01)$ indican un bajo nivel de dominancia dentro de la comunidad (cuadro 1). El parámetro $\alpha$ de Fisher resultó ser un buen estimador del número de especies representadas con un individuo por cuadro, ya que el valor de la constante $x$ calculado para cada cuadro nunca fue < 0.91 (Hayek y Buzas, 1997). En la mayoría de los casos el valor de $\alpha$ fue equivalente a por lo menos la cuarta parte del total de especies por cuadro, mientras que en otros (e.g. cuadro 3 ) representó poco más de $40 \%$. El valor promedio ( \pm 1 E.E.) calculado para el índice de Shannon fue $2.78 \pm 0.04$, y para su equitatividad fue $0.82 \pm 0.01$ (ambos calculados con $\ln$ ). 
José A. Gallardo-Cruz, Jorge A. Meave y Eduardo A. Pérez-García

Cuadro 1. Valores de los índices de diversidad y dominancia calculados para los 30 cuadros de muestreo del Cerro Verde, Nizanda, Oaxaca. $\mathrm{S}=$ Riqueza específica, $\mathrm{d}=$ Berger-Parker, $\mathrm{D}=$ Simpson, $\alpha=$ Alfa de Fisher, $\mathrm{H}^{\prime}=$ Shannon, $\mathrm{E}=$ Equitatividad .

\begin{tabular}{rrrrrrrrrrrrrr}
\hline Cuadro & $\mathrm{S}$ & $\mathrm{d}$ & $\mathrm{D}$ & $\alpha$ & $\mathrm{H}^{\prime}$ & $\mathrm{E}$ & Cuadro & $\mathrm{S}$ & $\mathrm{d}$ & $\mathrm{D}$ & $\alpha$ & $\mathrm{H}^{\prime}$ & $\mathrm{E}$ \\
\hline $\mathbf{1}$ & 36 & 0.20 & 0.07 & 11.60 & 3.01 & 0.84 & $\mathbf{1 6}$ & 36 & 0.16 & 0.07 & 11.40 & 2.94 & 0.82 \\
$\mathbf{2}$ & 27 & 0.14 & 0.06 & 11.55 & 2.90 & 0.88 & $\mathbf{1 7}$ & 29 & 0.12 & 0.07 & 10.14 & 2.85 & 0.85 \\
$\mathbf{3}$ & 25 & 0.15 & 0.06 & 10.77 & 2.86 & 0.89 & $\mathbf{1 8}$ & 34 & 0.15 & 0.08 & 9.58 & 2.92 & 0.83 \\
$\mathbf{4}$ & 31 & 0.19 & 0.08 & 9.62 & 2.84 & 0.83 & $\mathbf{1 9}$ & 32 & 0.13 & 0.06 & 10.08 & 3.02 & 0.87 \\
$\mathbf{5}$ & 33 & 0.15 & 0.07 & 9.69 & 2.90 & 0.83 & $\mathbf{2 0}$ & 32 & 0.21 & 0.07 & 9.95 & 3.00 & 0.86 \\
$\mathbf{6}$ & 31 & 0.29 & 0.11 & 11.73 & 2.79 & 0.81 & $\mathbf{2 1}$ & 27 & 0.20 & 0.11 & 7.94 & 2.60 & 0.79 \\
$\mathbf{7}$ & 30 & 0.34 & 0.15 & 9.92 & 2.51 & 0.74 & $\mathbf{2 2}$ & 39 & 0.14 & 0.06 & 12.60 & 3.17 & 0.87 \\
$\mathbf{8}$ & 35 & 0.25 & 0.12 & 9.74 & 2.68 & 0.75 & $\mathbf{2 3}$ & 27 & 0.17 & 0.09 & 7.80 & 2.68 & 0.81 \\
$\mathbf{9}$ & 24 & 0.13 & 0.08 & 6.56 & 2.70 & 0.85 & $\mathbf{2 4}$ & 31 & 0.17 & 0.08 & 10.15 & 2.87 & 0.83 \\
$\mathbf{1 0}$ & 28 & 0.20 & 0.10 & 8.05 & 2.63 & 0.79 & $\mathbf{2 5}$ & 31 & 0.15 & 0.06 & 11.61 & 2.97 & 0.87 \\
$\mathbf{1 1}$ & 24 & 0.12 & 0.06 & 8.94 & 2.83 & 0.89 & $\mathbf{2 6}$ & 30 & 0.17 & 0.07 & 7.48 & 2.96 & 0.87 \\
$\mathbf{1 2}$ & 23 & 0.40 & 0.19 & 6.57 & 2.26 & 0.72 & $\mathbf{2 7}$ & 34 & 0.32 & 0.15 & 10.68 & 2.51 & 0.71 \\
$\mathbf{1 3}$ & 24 & 0.41 & 0.22 & 5.23 & 2.12 & 0.67 & $\mathbf{2 8}$ & 32 & 0.23 & 0.11 & 9.19 & 2.64 & 0.76 \\
$\mathbf{1 4}$ & 33 & 0.25 & 0.10 & 9.06 & 2.84 & 0.81 & $\mathbf{2 9}$ & 21 & 0.15 & 0.08 & 7.70 & 2.68 & 0.88 \\
$\mathbf{1 5}$ & 22 & 0.14 & 0.08 & 6.17 & 2.73 & 0.88 & $\mathbf{3 0}$ & 39 & 0.21 & 0.07 & 12.62 & 3.16 & 0.86 \\
\hline
\end{tabular}

Estructura cuantitativa. Se registraron 1,478 individuos en el estrato alto y 1,141 en el bajo. A partir de esta última cifra se estimó que en el área muestreada ( 0.3 ha) existe un total de 5,705 individuos en el estrato bajo (cuadro 2). Las plantas del estrato bajo, cuya estatura promedio fue de $0.96 \mathrm{~cm}$, hicieron el mayor aporte a la densidad total del sistema y representaron $19.3 \%$ de la cobertura total. A su vez, el estrato alto (plantas con DAP $\geq 1 \mathrm{~cm}$ ), cuya altura promedio es $4.1 \mathrm{~m}$, fue mucho menos denso que el bajo, y aportó poco más de $80 \%$ de la cobertura sumada de los dos estratos. Los 83 árboles del estrato alto con $\mathrm{DAP} \geq 20 \mathrm{~cm}$ ( $1.1 \%$ del total; altura promedio $9.4 \mathrm{~m}$ ), aportaron $66.4 \%$ del área basal total y la cuarta parte de la cobertura total $(24.7 \%)$. La policaulescencia se registró con mayor frecuencia en los individuos con DAP de entre 1 y $5 \mathrm{~cm}$. El cuadro 2 contiene la síntesis estructural para otros conjuntos de plantas definidos por distintos límites inferiores de DAP.

La densidad total (estrato bajo + estrato alto) abarcó un intervalo de 9,900 a 51,000 ind. ha ${ }^{-1}$. La media ( \pm 1 E.E.) para esta variable fue de $23,950 \pm 1,643$ ind. ha' ${ }^{-1}$. A diferencia de la densidad total, el estrato alto hizo la mayor contribución a la cobertura total de la comunidad. En promedio, la cobertura total por cuadro fue de $44,674 \mathrm{~m}^{2} \mathrm{ha}^{-1}$, con un coeficiente de variación de $21.3 \%$. El intervalo para el área basal comprendió desde valores tan bajos como $17 \mathrm{~m}^{2}$ $\mathrm{ha}^{-1}$, hasta otros tan grandes como $297 \mathrm{~m}^{2} \mathrm{ha}^{-1}$ (promedio $=$ $\left.53 \pm 9.7 \mathrm{~m}^{2} \mathrm{ha}^{-1}\right)$. En uno de los cuadros con mayor área basal (cuadro 3), $88.5 \%$ del valor correspondió a un individuo de Beaucarnea stricta, cuyo DAP midió $1.83 \mathrm{~m}$.

El promedio por cuadro ( \pm 1 E.E.) de la variable "altura media" fue $4.1 \pm 0.1 \mathrm{~m}$. La variación de esta cifra fue menor (C.V. $=14.1 \%$; intervalo $=2.35-5.50)$ respecto al promedio de la "altura $10 \%$ superior" (promedio $=9.06$; C.V. $=18.5 \%$; intervalo $=5.72-13.62 \mathrm{~m}$ ).
Cuadro 2. Síntesis estructural por clase diamétrica para la selva baja caducifolia del Cerro Verde, Nizanda, Oaxaca. Las cifras corresponden a un área de 0.30 ha, y entre paréntesis se indican sus equivalentes porcentuales. Los valores del primer renglón (Total) representan la suma de las variables estructurales de los estratos alto y bajo.

\begin{tabular}{|c|c|c|c|c|c|}
\hline $\begin{array}{c}\text { Clase } \\
\text { diamétrica } \\
(\mathrm{cm})\end{array}$ & $\begin{array}{c}\text { No. } \\
\text { individuos }\end{array}$ & $\begin{array}{l}\text { No. } \\
\text { tallos }\end{array}$ & $\begin{array}{c}\text { Área basal } \\
\qquad\left(\mathrm{m}^{2}\right)\end{array}$ & $\begin{array}{c}\text { Cobertura } \\
\left(\mathrm{m}^{2}\right)\end{array}$ & $\begin{array}{c}\text { Altura } \\
\text { promedio } \\
(\mathrm{m})\end{array}$ \\
\hline Total & $\begin{array}{l}7183 \\
(100)\end{array}$ & $\begin{array}{l}8107 \\
(100)\end{array}$ & $\begin{array}{l}15.96 \\
(100)\end{array}$ & $\begin{array}{c}13327 \\
(100)\end{array}$ & - \\
\hline$<1$ & $\begin{array}{l}5705 \\
(79.4)\end{array}$ & $\begin{array}{l}5705 \\
(70.3)\end{array}$ & - & $\begin{array}{l}2581 \\
(19.3)\end{array}$ & 0.96 \\
\hline$\geq 1$ & $\begin{array}{l}1478 \\
(20.5)\end{array}$ & $\begin{array}{c}2402 \\
(29.6)\end{array}$ & $\begin{array}{l}15.96 \\
(100)\end{array}$ & $\begin{array}{l}10746 \\
(80.6)\end{array}$ & 4.13 \\
\hline$\geq 5$ & $\begin{array}{l}540 \\
(7.5)\end{array}$ & $\begin{array}{l}751 \\
(9.2)\end{array}$ & $\begin{array}{l}15.28 \\
(95.7)\end{array}$ & $\begin{array}{l}8018 \\
(60.1)\end{array}$ & 6.05 \\
\hline$\geq 10$ & $\begin{array}{l}249 \\
(3.4)\end{array}$ & $\begin{array}{l}263 \\
(3.2)\end{array}$ & $\begin{array}{l}13.51 \\
(84.6)\end{array}$ & $\begin{array}{l}5963 \\
(44.7)\end{array}$ & 7.74 \\
\hline$\geq 15$ & $\begin{array}{l}139 \\
(1.9)\end{array}$ & $\begin{array}{l}142 \\
(1.7)\end{array}$ & $\begin{array}{l}12.01 \\
(75.2)\end{array}$ & $\begin{array}{l}4368 \\
(32.7)\end{array}$ & 8.56 \\
\hline$\geq 20$ & $\begin{array}{c}83 \\
(1.1)\end{array}$ & $\begin{array}{c}83 \\
(1.0)\end{array}$ & $\begin{array}{l}10.61 \\
(66.4)\end{array}$ & $\begin{array}{l}3301 \\
(24.7)\end{array}$ & 9.39 \\
\hline
\end{tabular}

\section{Discusión}

Composición y diversidad florística. El Cerro Verde ocupa ca. $4 \%$ de los $85 \mathrm{~km}^{2}$ definidos por Pérez-García et al. (2001) como la región de Nizanda. La cifra de 194 especies registradas en este muestreo de vegetación no puede compararse directamente con otras publicadas para la región (Pérez-García et al., 2001), porque tales recuentos 
ESTRUCTURA Y DIVERSIDAD DE LA SElVA BAJA CADUCIFOLIA DEl CERRo Verde, OAXACA

Cuadro 3. Valores calculados para la estructura cuantitativa de los 30 cuadros de muestreo del Cerro Verde, Nizanda, Oaxaca. Los valores máximos y mínimos se indican subrayados y con negritas, respectivamente. E.E. = error estándar, C.V. = coeficiente de variación.

\begin{tabular}{|c|c|c|c|c|c|c|c|c|c|}
\hline \multirow[t]{2}{*}{ Cuadro } & \multicolumn{3}{|c|}{$\begin{array}{l}\text { Densidad } \\
(\text { ind. ha-1) }\end{array}$} & \multicolumn{3}{|c|}{$\begin{array}{c}\text { Cobertura } \\
\left(\mathrm{m}^{2} \mathrm{ha}^{-1}\right)\end{array}$} & \multirow[t]{2}{*}{$\begin{array}{l}\text { Área basal } \\
\left(\mathrm{m}^{2} \mathrm{ha}^{-1}\right)\end{array}$} & \multicolumn{2}{|c|}{$\begin{array}{c}\text { Altura } \\
(\mathrm{m})\end{array}$} \\
\hline & $\mathrm{DAP}<1 \mathrm{~cm}$ & $\mathrm{DAP}>1 \mathrm{~cm}$ & Total & $\mathrm{DAP}<1 \mathrm{~cm}$ & $\mathrm{DAP}>1 \mathrm{~cm}$ & Total & & Media & $10 \%$ Superior \\
\hline 1 & 19,500 & 5,200 & 24,700 & 11,479 & 24,192 & 35,672 & 17 & 3.70 & 9.26 \\
\hline 2 & 6,500 & 4,300 & 10,800 & 2,792 & 47,860 & 50,652 & 33 & 4.46 & 12.75 \\
\hline 3 & 7,000 & 2,900 & 9,900 & 4,700 & 43,930 & 48,630 & 128 & 4.73 & 10.30 \\
\hline 4 & 18,500 & 4,700 & 23,200 & 11,400 & 41,927 & 53,327 & 36 & 4.48 & 10.26 \\
\hline 5 & 22,000 & 6,200 & 28,200 & 8,720 & 35,581 & 44,301 & 37 & 3.74 & 9.38 \\
\hline 6 & 7,000 & 8,300 & 15,300 & 1,720 & $\underline{60,050}$ & $\underline{61,770}$ & 36 & 4.04 & 9.06 \\
\hline 7 & 14,000 & 5,400 & 19,400 & 4,416 & $\overline{29,520}$ & $\overline{33,936}$ & 60 & 3.63 & 7.66 \\
\hline 8 & 29,500 & 4,900 & 34,400 & 16,396 & 37,662 & 54,058 & 54 & 4.39 & 9.10 \\
\hline 9 & 19,500 & 5,300 & 24,800 & 10,200 & 29,568 & 39,768 & 53 & 3.86 & 8.58 \\
\hline 10 & 20,000 & 5,300 & 25,300 & 6,807 & 25,167 & 31,974 & 17 & 3.45 & 6.67 \\
\hline 11 & 7,500 & 4,700 & 12,200 & 5,874 & 39,404 & 45,278 & 54 & 4.20 & 9.58 \\
\hline 12 & 18,000 & 3,100 & 21,100 & 11,091 & 31,476 & 42,566 & 31 & 4.22 & 8.50 \\
\hline 13 & $\underline{44,500}$ & 6,500 & $\underline{51,000}$ & 9,636 & 29,972 & 39,608 & 29 & 4.01 & 8.58 \\
\hline 14 & $\overline{29,500}$ & 4,200 & $\overline{33,700}$ & 9,362 & 36,853 & 46,215 & 26 & 4.25 & 7.93 \\
\hline 15 & 16,500 & 4,700 & 21,200 & 6,257 & 15,857 & 22,114 & 30 & 3.57 & 7.96 \\
\hline 16 & 20,500 & 5,200 & 25,700 & 10,648 & 37,428 & 48,076 & 46 & 4.56 & 8.56 \\
\hline 17 & 12,000 & 4,700 & 16,700 & 6,444 & 29,132 & 35,576 & $\underline{297}$ & 2.35 & 7.86 \\
\hline 18 & 28,000 & 4,400 & 32,400 & 6,801 & 36,066 & 42,868 & $\overline{41}$ & 4.54 & 10.50 \\
\hline 19 & 18,000 & 5,100 & 23,100 & 7,312 & 32,080 & 39,393 & 45 & 3.88 & 9.76 \\
\hline 20 & 18,500 & 5,300 & 23,800 & 5,283 & 47,948 & 53,230 & 102 & $\underline{5.50}$ & 13.62 \\
\hline 21 & 19,500 & 3,500 & 23,000 & 11,889 & 49,656 & 61,546 & 65 & $\overline{4.13}$ & 9.50 \\
\hline 22 & 20,500 & 6,100 & 26,600 & 11,934 & 32,352 & 44,286 & 35 & 3.68 & 7.47 \\
\hline 23 & 20,000 & 4,100 & 24,100 & 16,508 & 43,240 & 59,748 & 56 & 4.15 & 11.95 \\
\hline 24 & 16,500 & 4,000 & 20,500 & 3,230 & 29,439 & 32,669 & 81 & 3.99 & 9.30 \\
\hline 25 & 9,500 & 6,100 & 15,600 & 2,487 & 35,393 & 37,881 & 30 & 3.90 & 7.93 \\
\hline 26 & 32,000 & $\underline{8,600}$ & 40,600 & 14,761 & 37,430 & 52,191 & 47 & 3.74 & 6.92 \\
\hline 27 & 21,000 & 3,700 & 24,700 & 6,368 & 29,467 & 35,836 & 20 & 4.80 & 9.25 \\
\hline 28 & 25,500 & 3,500 & 29,000 & $\underline{21,711}$ & 35,654 & 57,366 & 40 & 4.11 & 8.24 \\
\hline 29 & 7,500 & 3,500 & 11,000 & 4,369 & 37,495 & 41,865 & 20 & 3.01 & 5.72 \\
\hline 30 & 22,000 & 4,500 & 26,500 & 7,522 & 40,288 & 47,811 & 32 & 4.30 & 9.52 \\
\hline Promedio & $19,017.7$ & 4,933.3 & $23,950.0$ & $8,604.0$ & $36,070.7$ & $44,674.6$ & 53.2 & 4.05 & 9.06 \\
\hline E.E. & $1,572.7$ & 245.6 & 1,643.7 & 863.2 & $1,618.6$ & $1,773.3$ & 9.7 & 0.1 & 0.3 \\
\hline C.V. (\%) & 44.5 & 26.8 & 36.9 & 54.0 & 24.1 & 21.3 & 97.9 & 14.1 & 18.5 \\
\hline
\end{tabular}

han excluido sistemáticamente a las entidades no determinadas hasta el nivel específico. Sin embargo, cabe destacar que las 141 especies cuyos epítetos específicos pudieron ser conocidos equivalen a más de la tercera parte de la cifra de 450 especies, que están en la misma situación, actualmente citada para la SBC regional (Pérez-García et al., en prensa).

El número de familias registradas en el estudio (52) es casi igual al reportado para la vegetación propia de los afloramientos de roca caliza presentes en Nizanda (54 familias; Pérez-García y Meave, 2004), y representa $70 \%$ de las familias ubicadas en la comunidad de SBC de la región (Pérez-García et al., 2001). En general, las familias más diversas se encuentran entre las más comunes en las selvas secas mexicanas y del Neotrópico (Gentry, 1995; Trejo, 1998). Las dos familias más ricas en especies, Leguminosae y Asteraceae, comprenden en conjunto $24.2 \%$ del total de especies registradas en el muestreo. A ellas les siguen Euphorbiaceae, Apocynaceae, Malpighiaceae, Rubiaceae, Bignoniaceae, Capparaceae y Solanaceae. Todas éstas, incluyendo las dos primeras, se ubican entre las familias más diversas ( $\geq 5$ especies) en los enclaves de vegetación xerofítica de Nizanda (PérezGarcía, 2002), y en las localidades de selvas secas analizadas por Gentry (1995). La segunda posición ocupada por Asteraceae parecería constituir una peculiaridad de esta selva, ya que en ninguno de los muestreos realizados por Gentry (1995) esta familia ocupa una posición tan alta. Sin embargo, es más probable que esta discrepancia sea producto de los diferentes criterios utilizados en los muestreos de vegetación. Por ejemplo, en el trabajo realizado por Gentry (1988) en Capeira, Ecuador, Asteraceae no figura entre las familias importantes, a pesar de que en la flórula de dicha localidad es la segunda familia más rica en especies (Dodson y Gentry, 1992).

Al igual que en otras selvas secas de México, en este 
estudio no se observó una correspondencia clara entre la riqueza de especies por familia y la abundancia de individuos en la comunidad (Lott et al., 1987; Trejo, 1998). Si bien algunas familias fueron ricas y abundantes a la vez (Leguminosae, Asteraceae y Euphorbiaceae), esto no fue siempre así: ciertas familias típicas de este tipo de ambientes o de zonas áridas y semiáridas, como Burseraceae y Cactaceae (Gentry, 1995; Rzedowski et al., 2004, 2005), no se encontraron entre las más diversas en el Cerro Verde, pero algunos de sus elementos fueron muy abundantes y frecuentes (e.g. Bursera spp. y Pilosocereus collinsii).

La comparación de la riqueza específica de la SBC del Cerro Verde con la de otras comunidades vegetales presentes en la misma región es posible debido a que para todas ellas se cuenta con muestras equivalentes (parcelas de $100 \mathrm{~m}^{2}$ ). Esta comparación indicó que en términos de la riqueza promedio por parcela, la SBC del Cerro Verde tiene una densidad de especies intermedia, por debajo del matorral espinoso, la vegetación ribereña y la SBC desarrollada sobre suelos profundos, fuera del Cerro Verde, pero mayor que la densidad de la sabana, del matorral inerme, la SBCr y del matorral xerófilo (cuadro 4; Meave y PérezGarcía, 2000).

Si bien la densidad de especies en el Cerro Verde fue superada por los valores de algunas comunidades de Nizanda, su valor promedio del índice de Shannon para el estrato alto $\left(\mathrm{H}^{\prime}=2.78\right)$ fue semejante al reportado por Pérez-García y Meave (2004) para la SBC en suelo presente en otros afloramientos calcáreos de la región $\left(\mathrm{H}^{\prime}=\right.$ 2.89) y mucho mayor que el calculado para la vegetación ribereña $\left(H^{\prime}=1.22\right.$; Lebrija-Trejos, 2001). Es decir, su diversidad ecológica es en realidad alta. Los diferentes índices de dominancia y diversidad calculados (Simpson,

Cuadro 4. Riqueza de especies en ocho comunidades vegetales de la región de Nizanda, Oaxaca. EVX = enclaves de vegetación xerofíti$\mathrm{ca}, \mathrm{ME}=$ matorral espinoso, $\mathrm{MI}=$ matorral inerme, $\mathrm{MX}=$ matorral xerófilo, SBCs = selva baja caducifolia en suelo desarrollado, $\mathrm{SBCr}=$ selva baja en roca caliza expuesta, $V R=$ vegetación ribereña, $N=$ número total de muestras, E.E. = error estándar. Los promedios expresan el número de especies por $100 \mathrm{~m}^{2}$.

\begin{tabular}{lccc}
\hline Comunidad & $N$ & Promedio & E.E. \\
\hline Sabana ${ }^{1}$ & 39 & 28.3 & 0.6 \\
ME $^{1}$ & 21 & 41.4 & 1.4 \\
MI $^{1}$ & 8 & 28.3 & 1.2 \\
VR $^{2}$ & 26 & 37.3 & 2.7 \\
EVX $^{3}$ & & & \\
$\quad$ SBCs & 9 & 36.9 & 1.5 \\
$\quad$ SBCr & 9 & 25.3 & 3.3 \\
$\quad$ MX & 9 & 11.6 & 0.9 \\
CerroVerde & 30 & 30.0 & 0.9 \\
\hline
\end{tabular}

' Meave y Pérez-García (2000)

${ }^{2}$ Lebrija-Trejos (2001)

${ }^{3}$ Pérez-García y Meave (2004)
Berger-Parker, $\alpha$ de Fisher y equitatividad) refuerzan esta conclusión. No obstante, el índice de Shannon promedio calculado en el Cerro Verde fue menor que el reportado por Lott et al. (1987) para transectos de $50 \times 2 \mathrm{~m}$ en Chamela $\left(\mathrm{H}^{\prime}=3.84\right)$. Cabe aclarar que la cifra reportada por estos últimos autores (5.75) fue obtenida usando logaritmos de base 2; el valor aquí citado se obtuvo transformándola con la fórmula de equivalencia presentada por Cox (1996). Estas comparaciones deben, en todo caso, tomarse con extrema cautela, ya que los conjuntos comparados suelen no ser enteramente equivalentes. Por ejemplo, la cifra de Chamela corresponde a individuos con DAP $\geq 2.5 \mathrm{~cm}$, lo cual podría resultar en un valor más alto para Chamela como resultado de esta particularidad metodológica.

El análisis del aporte relativo de cada estrato (alto y bajo) a la riqueza total del sistema mostró una fuerte equivalencia entre ambos. El número de especies exclusivas de cada uno de ellos fue idéntico (49;25.3\% del total), y el número de especies compartidas (96) equivalió casi exactamente a la mitad de las especies registradas. Esta diferenciación florística entre estratos no se limitó al nivel de especie, sino que también se observó para taxa superiores. Las especies de las familias Achatocarpaceae, Bombacaceae, Buxaceae, Caricaceae, Convolvulaceae, Julianiaceae, Moraceae, Nolinaceae, Sapotaceae y Zygophyllaceae sólo aparecieron en el estrato alto, mientras que Araceae, Bromeliaceae, Commelinaceae, Phytolacaceae, Poaceae, Portulacaceae y Sterculiaceae fueron exclusivas del bajo. La relativa independencia de la composición de especies de cada estrato acentúa el carácter aditivo en la determinación de la riqueza total de la comunidad. Otros estudios en comunidades similares han sugerido patrones análogos en la estratificación de la riqueza (e.g. Gentry y Emmons, 1987). Sin embargo, la ausencia de información cuantitativa en la mayoría de los estudios desarrollados en selvas secas sobre la fracción vegetal definida aquí como estrato bajo impide realizar comparaciones más directas.

Estructura cuantitativa. La fisonomía de la comunidad está determinada fundamentalmente por un reducido número de individuos con DAP $\geq 5 \mathrm{~cm}$. No obstante, en términos de densidad y número de tallos, los elementos con diámetros inferiores constituyen una porción grande en la conformación de la estructura comunitaria, ya que representan $90 \%$ de la densidad total.

La densidad promedio del estrato alto en el Cerro Verde fue menor que la reportada para la comunidad de SBC asentada en suelos profundos de los afloramientos calizos de Nizanda (Pérez-García y Meave, 2004), pero mayor que la citada para la vegetación ribereña (Lebrija-Trejos, 2001). Lamentablemente, las diferencias metodológicas respecto a otros estudios, sobre todo en los criterios de inclusión y la forma o el área de las unidades de muestreo, limitan el 


\section{Estructura y diVersidad de la SElVa baja CADUCIFOlia del CerRo Verde, OAXaCA}

Cuadro 5. Atributos estructurales para diferentes tipos de vegetación tropical. $\mathrm{P}=$ precipitación anual promedio, $\mathrm{T}=$ temperatura media anual. Unidad de muestreo: parcela $(\mathrm{m})$ o $\mathrm{CCP}=$ cuadrantes centrados en un punto. $\mathrm{N}=$ número total de muestras. DAP indica el límite inferior considerado en las mediciones. BTS = bosque tropical subperennifolio, $\mathrm{EVX}=$ enclaves de vegetación xerofítica, $\mathrm{SBC}=$ selva baja caducifolia, $\mathrm{VR}=$ vegetación ribereña.

\begin{tabular}{|c|c|c|c|c|c|c|c|c|}
\hline Localidad / Clasificación & $\begin{array}{c}\text { Clima } \\
\left(\mathrm{P}=\mathrm{mm}, \mathrm{T}={ }^{\circ} \mathrm{C}\right)\end{array}$ & $\begin{array}{l}\text { Altitud } \\
\text { (m snm) }\end{array}$ & $\begin{array}{l}\text { Unidad de } \\
\text { muestreo }\end{array}$ & N & $\begin{array}{l}\text { DAP } \\
(\mathrm{cm})\end{array}$ & $\begin{array}{l}\text { Densidad } \\
\text { (ind. ha') }\end{array}$ & $\begin{array}{l}\text { Área basal } \\
\left(\mathrm{m}^{2} \text { ha- }^{-1}\right)\end{array}$ & Fuente \\
\hline $\begin{array}{l}\text { San Javier, Sonora } \\
\left(28^{\circ} 36^{\prime} \mathrm{N}, 109^{\circ} 44^{\prime} \mathrm{O}\right)\end{array}$ & $\begin{array}{l}P=638 \\
T=18.7\end{array}$ & & & & & & & Martínez-Yrízar et al. (2000) \\
\hline San Juan I, SBC & & 1,000 & $50 \times 2$ & 16 & $\geq 3.0$ & 1550 & 20.1 & \\
\hline San Juan II, SBC & & 900 & $\mathrm{CCP}$ & 22 & $\geq 3.0$ & 2120 & 28.1 & \\
\hline Corrales, SBC & & 550 & $50 \times 2$ & 16 & $\geq 3.0$ & 1425 & 26.7 & \\
\hline Mirador, SBC & & 800 & $50 \times 2$ & 16 & $\geq 3.0$ & 1813 & 17.5 & \\
\hline $\begin{array}{l}\text { Chamela, Jalisco } \\
\left(19^{\circ} 30^{\prime} \mathrm{N}, 105^{\circ} 03^{\prime} \mathrm{O}\right)\end{array}$ & $\begin{array}{l}P=748 \\
T=24.9\end{array}$ & & & & & & & \\
\hline Lomeríos y Arroyos. & & & & & & & & Lott et al. (1987) \\
\hline Lomerío I, SBC & & - & $50 \times 2$ & 10 & $\geq 2.5$ & 3940 & 26.1 & \\
\hline Lomerío II, SBC & & - & $50 \times 2$ & 10 & $\geq 2.5$ & 5060 & 21.8 & \\
\hline Arroyos, BTS & & - & $50 \times 2$ & 10 & $\geq 2.5$ & 4520 & 52.4 & \\
\hline Cuenca. & & & & & & & & Martínez-Yrízar et al. (1996) \\
\hline Arriba, SBC & & 150 & $80 \times 40$ & 1 & $\geq 3.18$ & 2790 & 12.7 & \\
\hline Medio, SBC & & 130 & $80 \times 40$ & 1 & $\geq 3.18$ & 3221 & 17.3 & \\
\hline Abajo, SBC & & 70 & $80 \times 40$ & 1 & $\geq 3.18$ & 2104 & 19.8 & \\
\hline Lomerío, SBC & & 90 & $50 \times 20$ & 1 & $\geq 3.0$ & 2140 & 25.6 & Martínez-Yrízar et al. (1992) \\
\hline $\begin{array}{l}\text { Álamos, Sonora } \\
\left(27^{\circ} 01^{\prime} \mathrm{N}, 108^{\circ} 56^{\prime} \mathrm{O}\right)\end{array}$ & $\begin{array}{l}P=664 \\
T=23.8\end{array}$ & & & & & & & Martínez-Yrízar et al. (2000) \\
\hline Vara Blanca, SBC & & - & СCP & 16 & $\geq 3.0$ & 1702 & 20.7 & \\
\hline Tempisque, SBC & & 500 & СCP & 29 & $\geq 3.0$ & 1772 & 15.4 & \\
\hline La Luna, SBC & & 450 & CCP & 46 & $\geq 3.0$ & 1900 & 40.4 & \\
\hline $\begin{array}{l}\text { Región Costa Oaxaca, SBC } \\
\left(15^{\circ} 50^{\prime} \mathrm{N}, 95^{\circ} 59^{\prime} \mathrm{O}\right)\end{array}$ & $\begin{array}{c}P=812-961 \\
T=25.0\end{array}$ & $0-1050$ & $50 \times 2$ & 15 & $\geq 2.5$ & 3050 & 23.4 & Salas-Morales (2002) \\
\hline $\begin{array}{l}\text { Cosalá, Sinaloa, SBC } \\
\left(24^{\circ} 30^{\prime} \mathrm{N}, 106^{\circ} 45^{\prime} \mathrm{O}\right)\end{array}$ & $\begin{array}{l}\mathrm{P}=900 \\
\mathrm{~T}=24.5\end{array}$ & 561 & $50 \times 2$ & 10 & $\geq 1.0$ & 5170 & 62.1 & Trejo (1998) \\
\hline $\begin{array}{l}\text { Caleta, Michoacán, SBC } \\
\left(18^{\circ} 07^{\prime} \mathrm{N}, 102^{\circ} 52^{\prime} \mathrm{O}\right)\end{array}$ & $\begin{array}{l}P=1200 \\
T=24.5\end{array}$ & 97 & $50 \times 2$ & 10 & $\geq 1.0$ & 7770 & 79.1 & Trejo (1998) \\
\hline $\begin{array}{l}\text { Tehuantepec, Oax., SBC } \\
\left(16^{\circ} 21^{\prime} \mathrm{N}, 95^{\circ} 24^{\prime} \mathrm{O}\right)\end{array}$ & $\begin{array}{l}\mathrm{P}=920 \\
\mathrm{~T}=26.5\end{array}$ & 274 & $50 \times 2$ & 10 & $\geq 1.0$ & 5320 & 32.3 & Trejo (1998) \\
\hline $\begin{array}{l}\text { Cerro Tuxpan, Gro., SBC } \\
\left(18^{\circ} 24^{\prime} N, 99^{\circ} 29^{\prime} \mathrm{O}\right)\end{array}$ & $\begin{array}{l}P=1050 \\
T=22.9\end{array}$ & 1259 & $50 \times 2$ & 10 & $\geq 1.0$ & 5770 & 52.7 & Trejo (1998) \\
\hline $\begin{array}{l}\text { Trinitaria, Chiapas, SBC } \\
\left(16^{\circ} 01^{\prime} \mathrm{N}, 92^{\circ} 01^{\prime} \mathrm{O}\right)\end{array}$ & $\begin{array}{l}P=1000 \\
T=24.0\end{array}$ & 890 & $50 \times 2$ & 10 & $\geq 1.0$ & 6260 & 46.5 & Trejo (1998) \\
\hline $\begin{array}{l}\text { Sayil, Yucatán, SBC } \\
\left(20^{\circ} 10^{\prime} \mathrm{N}, 89^{\circ} 38^{\prime} \mathrm{O}\right)\end{array}$ & $\begin{array}{l}\mathrm{P}=932 \\
\mathrm{~T}=26.5\end{array}$ & 100 & $50 \times 2$ & 10 & $\geq 1.0$ & 7290 & 36.7 & Trejo (1998) \\
\hline $\begin{array}{l}\text { Nizanda, Oaxaca } \\
\left(16^{\circ} 39^{\prime} \mathrm{N}, 95^{\circ} 00^{\prime} \mathrm{O}\right)\end{array}$ & $\begin{array}{l}P=1000 \\
T=25.0\end{array}$ & & & & & & & \\
\hline EVX - SBC & & $100-250$ & $10 \times 10$ & 9 & $\geq 1.0$ & 5510 & 31.9 & Pérez-García (2002) \\
\hline VR & & $90-150$ & $20 \times 5$ & 26 & $\geq 1.0$ & 2865 & 71.0 & Lebrija-Trejos (2001) \\
\hline Cerro Verde, SBC & & $100-430$ & $10 \times 10$ & 30 & $\geq 1.0$ & 4933 & 53.0 & Este trabajo \\
\hline SBC, promedio & - & - & $50 \times 2$ & 200 & $\geq 1.0$ & 5820 & 56.0 & Trejo (1998) \\
\hline
\end{tabular}


José A. Gallardo-Cruz, Jorge A. Meave y Eduardo A. Pérez-García

número de comparaciones directas de esta variable estructural con otras selvas secas de México. Las pocas que fueron posibles, por ejemplo con los datos reportados por Trejo (1998) y Gentry (1995) para varios sitios de México y otros países, sugieren que la densidad promedio de la SBC del Cerro Verde queda dentro del intervalo reconocido para estas comunidades (cuadro 5).

Un aspecto notable de la estructura de esta selva es la abundancia de individuos policaulescentes. Éstos no estuvieron igualmente representados en todas las clases diamétricas, sino que se concentraron en las plantas de entre 1 y $10 \mathrm{~cm}$ de DAP. La policaulescencia es un fenómeno frecuente no sólo en las selvas secas, sino en varios tipos de vegetación, por ejemplo en los bosques montanos húmedos, en los que se relaciona directamente con la altitud (Valencia y Jørgensen, 1992; Valencia, 1995). En el caso particular de los bosques tropicales y subtropicales de tierras bajas, la alta incidencia de la policaulescencia parece estar favorecida por ciertos tipos de disturbio (Dunphy et al., 2000), o por la alta frecuencia de la regeneración por rebrotes (Lebrija-Trejos, 2004).

A diferencia de las otras variables de diversidad y estructura discutidas hasta aquí, los valores de área basal obtenidos en el Cerro Verde son más altos que los reportados para la mayoría de las otras comunidades vegetales comparadas. Solamente los registrados para la vegetación ribereña y la SBCr de Nizanda, así como para las SBC de Cosalá (Sinaloa) y Caleta (Michoacán) descritas por Trejo (1998) son más grandes (cuadro 5). De hecho, el valor calculado para el Cerro Verde rebasó el límite superior del intervalo global $\left(17-40 \mathrm{~m}^{2}\right)$ propuesto por Murphy y Lugo (1995) para las selvas de la zona ecológica en la que se ubica Nizanda. Asimismo, el valor promedio para el Cerro Verde es equivalente a los reportados para comunidades de bosques tropicales húmedos y subhúmedos en América (Meave y Kellman, 1994; Gentry, 1995; Valle-Doménech, 2000), en los que existen mucho menos restricciones para el crecimiento vegetal.

En el análisis comparativo de esta variable estructural, es importante intentar discernir los factores que determinan sus valores, ya que un valor de área basal muy elevado podría interpretarse como un indicio de una elevada productividad en la comunidad. Sin embargo, esta interpretación puede ser errónea. Por ejemplo, Pérez-García y Meave (2004) argumentan que el alto valor de área basal de la SBCr en esta localidad puede deberse a la abundancia de individuos policaulescentes o a la presencia de tallos suculentos adaptados al almacenamiento de agua. La policaulescencia no parece ser una explicación adecuada para el caso del Cerro Verde, ya que los individuos que la presentan tienen por lo general diámetros pequeños, lo que se refleja en un aporte moderado (15\%) al área basal total. En realidad, los responsables del alto valor de área basal fueron un reducido número de individuos de grandes dimensiones, particularmente en diámetro. Los cuadros 3 y 17 ejemplifican esto, en los que un solo individuo de Beaucarnea stricta y otro de Jacaratia mexicana, respectivamente, aportó cada uno más de $70 \%$ del área basal en su cuadro. Estas observaciones deben ser tomadas en cuenta al interpretar cifras de área basal en este tipo de comunidades, ya que la diferencia entre los valores calculados con y sin este tipo de plantas llegan a ser muy grandes. Cabe aclarar que este tipo de individuos de gran talla no son tan raros en la comunidad, e incluso existen porciones del cerro donde abundan. Por ello, el valor de área basal reportado en este estudio y su varianza asociada no deben verse como un artificio azaroso del muestreo, sino como una representación relativamente realista de la comunidad.

En promedio, la cobertura total del dosel superó $400 \%$. Este valor indica un sobrelapamiento considerable de las copas de los árboles que conforman esta comunidad. Desafortunadamente no se dispone de muchos datos sobre esta variable para otras comunidades similares. Sin embargo, este valor es consistente con el reportado para la SBC en suelo profundo asociada a los afloramientos calcáreos de Nizanda (Pérez-García y Meave, 2004).

Las variables empleadas para evaluar la estatura de la comunidad ("altura media" y " $10 \%$ superior") fueron muy homogéneas entre sitios. La "altura media", que tuvo un valor de poco más de $4 \mathrm{~m}$, es una medida de la estatura promedio de todas las plantas del estrato alto de la comunidad y por ello no refleja la altura de la parte alta del dosel; por lo tanto, es lógico que sea menor que el valor citado con anterioridad como la altura de la SBC de Nizanda $(\approx 7$ m; Pérez-García et al., 2001). Por el contrario, la variable " $10 \%$ superior" constituye una medida más indicativa del límite superior del dosel, ya que su cálculo considera solamente individuos que alcanzan dicho límite (SalasMorales, 2002). Se puede concluir entonces que la altura global de la SBC del Cerro Verde $(\approx 9 \mathrm{~m})$ rebasa notoriamente (en casi 30\%) la de la SBC establecida en otras zonas de Nizanda.

En síntesis, los resultados de este estudio muestran a la SBC del Cerro Verde como una comunidad típica de las selvas secas del sur de México y otras regiones neotropicales. Solamente el área basal parece rebasar de manera notable a los intervalos conocidos para este tipo de vegetación, lo cual sugiere que las particularidades ambientales de este sitio han seleccionado el desarrollo de la suculencia en la comunidad. Este rasgo estructural tendría que ser examinado con más detalle en futuras investigaciones, sobre todo bajo la óptica de las posibles restricciones ambientales (Robert y Moravie, 2003), entre ellas el efecto diferencial de la orientación y la altitud en la localidad (Gallardo-Cruz, 2004).

Consideraciones para la conservación. El Cerro Verde alberga posiblemente las poblaciones más numerosas de 
dos especies endémicas de la región de estudio: Agave nizandensis Cutak (Agavaceae) y Encyclia nizandensis Pérez-García et Hágsater (Orchidaceae). Llama la atención además un conjunto de especies enlistadas en la Norma Oficial Mexicana (NOM-059-ECOL-2001; Diario Oficial de la Federación, 2002) como endémicas de México y ubicadas en alguna categoría de riesgo: Beaucarnea stricta Lem. (Nolinaceae) y Tillandsia socialis L.B.Sm. (Bromeliaceae) están citadas en la categoría de amenazada, mientras que Barkeria whartoniana (C.Schweinf.) SotoArenas (Orchidaceae) y Peniocereus fosterianus Cutak (Cactaceae) están sujetas a protección especial. Asimismo, sin ser endémicas del país, otras especies también están en dicha Norma Oficial: Tillandsia concolor L.B.Sm. (Bromeliaceae) y Astronium graveolens Jacq. (Anacardiaceae) están enlistadas como amenazadas. Un caso particularmente interesante es el de Pleurothallis digitale Luer (Orchidaceae), especie clasificada como amenazada, que se trata de un taxón propio de la vertiente atlántica, de modo que las poblaciones de Nizanda representan el límite sur-occidental de su distribución geográfica.

Además, muchas especies conocidas de la flora de Nizanda sólo han sido recolectadas en el Cerro Verde; ejemplos de ello son Anthurium cerrobaulense Matuda (Araceae), Bauhinia cookii Rose y Lonchocarpus longipedicellatus Pittier (Leguminosae), Oldelandia microphylla Bremek. (Rubiaceae), y Prestonia mexicana A.DC. y Stemmadenia eubracteata Woodson (Apocynaceae), entre muchas otras. De hecho, el único representante de la familia Buxaceae, Buxus bartletti Standl., sólo se conoce del Cerro Verde. A esta lista deben añadirse las poblaciones de al menos tres especies nuevas para la ciencia, Amyris sp. (Rutaceae), Lonchocarpus sp. (Leguminosae) y Yucca sp. (Agavaceae), así como de Guaiacum coulteri A.Gray (Zygophyllaceae), que actualmente está considerada como una de las especies arbóreas con mayor riesgo en el país.

Los datos estructurales, en particular los de altura pero también los de área basal, denotan un buen estado de conservación en comparación con otros manchones de SBC en la zona de estudio. Esta situación puede deberse, al menos en parte, a la dificultad de realizar en él actividades agropecuarias y extractivas debido en gran parte a sus pendientes muy pronunciadas. Sin embargo, el aumento actual de la presión sobre la tierra podría producir nuevos impactos que lleven a su deterioro. La información presentada en este trabajo, junto con la obtenida previamente a partir de numerosas expediciones de exploración florística de esta localidad, justifican plenamente los esfuerzos de conservación del Cerro Verde por su alto valor biológico y los múltiples servicios ecosistémicos que seguramente brinda tanto a los pobladores de Nizanda como de la región en general.

Agradecimientos
Bartolino Reyes, Leticia Ochoa, Hugo Tovar y Martín Zurita colaboraron en el trabajo de campo, y Marco Antonio Romero apoyó en el trabajo de cómputo. Estamos agradecidos con los pobladores de Nizanda por el apoyo brindado para la realización de este trabajo, en particular con la generosidad de la familia Reyes Manuel. Guillermo Ibarra, Angelina Martínez e Irma Trejo hicieron valiosos comentarios sobre versiones previas del trabajo. Este estudio recibió financiamiento del Fondo Sectorial SEMARNAT-CONACYT (Proyecto C021-0287) y de la UNAM (PAPIIT, Proyecto IN221503). El primer autor agradece a ambas instituciones las becas otorgadas.

\section{Literatura citada}

Acosta-Castellanos S. 1995. Introducción a la fitogeografía de Oaxaca. En: Vázquez-Dávila M.A. Ed. Sociedad y Naturaleza en Oaxaca. La Tecnología Agrícola Tradicional. pp. 39-47, Instituto Indigenista Interamericano, CONACyT, e Instituto Tecnológico de Oaxaca, Oaxaca.

Anónimo. 1981. Atlas Nacional del Medio Físico. Secretaría de Programación y Presupuesto. México, D.F.

Anónimo. 1984a. Carta de efectos climáticos regionales mayooctubre. Juchitán E15-10 D15-1, escala 1:250,000. Secretaría de Programación y Presupuesto, México, D.F.

Anónimo. 1984b. Carta de efectos climáticos regionales noviembre-abril. Juchitán E15-10 D15-1, escala 1:250,000. Secretaría de Programación y Presupuesto, México, D.F.

Balvanera P., Lott E., Segura G., Siebe C. e Islas A. 2002. Patterns of $\beta$-diversity in a Mexican tropical dry forest. Journal of Vegetation Science 13:145-158.

Búrquez A., Martínez-Yrízar A., Felger R.S. y Yetman D. 1999. Vegetation and habitat diversity at the southern edge of the Sonoran desert. En: Robichaux R.H. Ed. Ecology of Sonoran desert plants and plant communities. The University of Arizona Press, Tucson.

Challenger A. 1998. Utilización y Conservación de los Ecosistemas Terrestres de México: Pasado, Presente y Futuro. Comisión Nacional para el Conocimiento y Uso de la Biodiversidad, Instituto de Biología, UNAM y Agrupación Sierra Madre, S.C., México, D.F.

Cox G.W. 1996. Laboratory Manual of General Ecology. 7a ed. Wm.C.Brown Publishers, Dubuque.

Cronquist A. 1981. An Integrated System of Classification of Flowering Plants. Columbia University Press, Nueva York.

Dahlgren R.M.T., Clifford H.T. y Yeo P.F. 1985. The Families of the Monocotyledons: Structure, Evolution, and Taxonomy. Springer-Verlag, Berlín.

Diario Oficial de la Federación. 2002. Norma Oficial Mexicana Nom-059-Ecol-2001, Protección Ambiental - Especies Nativas de México de Flora y Fauna Silvestres - Categorías de Riesgo y Especificaciones para su Inclusión, Exclusión o Cambio - Lista de Especies en Riesgo. Miércoles 6 de marzo de 2002, pp. 2-56.

Dodson C.H. y Gentry A.H. 1992. Flórula de Capeira. Banco Nacional de Ecuador, Quito. 
Dunphy B.K., Murphy P.G. y Lugo A.E. 2000. The tendency for trees to be multiple-stemmed in tropical and subtropical dry forests: studies of Guanica forest, Puerto Rico. Tropical Ecology 41:161-167.

Durán E., Balvanera P., Lott E., Segura G., Pérez-Jiménez A., Islas A. y Franco M. 2002. Estructura, composición y dinámica de la vegetación. En: Noguera F.A., Vega-Rivera J.H., García-Aldrete A.N. y Quesada-Avendaño M. Eds. Historia Natural de Chamela, pp. 443-472. Instituto de Biología, Universidad Nacional Autónoma de México, México, D.F.

Gallardo-Cruz J.A. 2004. Efecto de la orientación y la altitud sobre la heterogeneidad vegetacional en el Cerro Verde, Nizanda (Oaxaca), México. Tesis de Licenciatura (Biología), Facultad de Ciencias, Universidad Nacional Autónoma de México, México, D.F., 79 pp.

García E. 1988. Modificaciones al Sistema de Clasificación Climática de Köeppen (Para Adaptarlo a las Condiciones de la República Mexicana). Editado por la autora. $4^{\text {a }}$ ed. México, D.F.

Gentry A.H. 1988. Changes in plant community diversity and floristic composition on environmental and geographical gradients. Annals of the Missouri Botanical Garden 75:1-34.

Gentry A.H. 1995. Diversity and floristic composition of neotropical dry forest. En: Bullock S.H., Mooney H.A. y Medina E. Eds. Seasonally Dry Tropical Forests, pp. 146-194, Cambridge University Press, Cambridge.

Gentry A.H. y Emmons L.H. 1987. Geographical variation in fertility, phenology, and composition of the understory of neotropical forests. Biotropica 19:216-227.

Gentry H.S. 1942. Rio Mayo Plants: A Study of the Flora and Vegetation of the Valley of the Rio Mayo, Sonora. Carnegie Institution of Washington. Washington D.C.

Hayek L.C. y Buzas M.A. 1997. Surveying Natural Populations. Columbia University Press, Nueva York.

Lebrija-Trejos E. 2001. Análisis estructural de la vegetación ribereña en la región de Nizanda, Oaxaca, México. Tesis de Licenciatura, Facultad de Ciencias, Universidad Nacional Autónoma de México, México, D.F., 133 pp.

Lebrija-Trejos E. 2004. Secondary succesion in a tropical dry forest of southern Mexico. Tesis de Maestría, Universidad de Wageningen, Wageningen, $68 \mathrm{pp}$.

Lorence D.H. y García-Mendoza A. 1989. Oaxaca, Mexico. En: Campbell D.G. y Hammond H.D. Eds. Floristic Inventory of Tropical Countries: The Status of Plant Systematics, Collections, and Vegetation, Plus Recommendations for the Future. pp. 253-269. New York Botanical Garden. Nueva York.

Lott E.J., Bullock S.H. y Solís-Magallanes A. 1987. Floristic diversity structure of upland and arroyo forests of Coastal Jalisco. Biotropica 19:228-235.

Magurran A.E. 1988. Ecological Diversity and its Measurement. Princeton University Press, Princeton.

Martin P.S., Yetman D., Fishbein M., Jenkins P., van Devender T.R. y Wilson R.K. 1998. Gentry's Río Mayo Plants. The tropical deciduous forest and environs of northwest Mexico. University of Arizona Press, Tucson.

Martínez-Yrízar A., Búrquez A. y Maass M. 2000. Structure and functioning of tropical deciduous forest in Western Mexico. En: Robichaux R.H. y Yetman D.A. Eds. The Tropical Deciduous Forest of Alamos: Biodiversity of a Threatened
Ecosystem in Mexico, pp. 19-35, The University of Arizona Press, Tucson.

Martínez-Yrízar A., Maass J.M., Pérez-Jimenez L.A. y Sarukhán J. 1996. Net primary productivity of a tropical deciduous forest ecosystem in western Mexico. Journal of Tropical Ecology 12:169-175.

Martínez-Yrízar A., Sarukhán J., Pérez-Jiménez A., Rincón E., Maass J.M., Solís-Magallanes A. y Cervantes L. 1992. Aboveground phytomass of a tropical deciduous forest on the coast of Jalisco, México. Journal of Tropical Ecology 8:87-96.

Matteucci S.D. y Colma A. 1982. Metodología para el Estudio de la Vegetación. Organización de los Estados Americanos, Washington, D.C.

Meave J. y Kellman M. 1994. Maintenance of rain forest plant diversity in tropical riparian forest fragments: implications for species conservation during Pleistocene drought. Journal of Biogeography 21:121-135.

Meave J. y Pérez-García E.A. 2000. Estudio de la diversidad florística de la región de Nizanda, Istmo de Tehuantepec, Oaxaca (Proyecto L085). Informe final inédito presentado a la CONABIO. Facultad de Ciencias, Universidad Nacional Autónoma de México, México, D.F., 41 pp.

Miranda F. 1942. Estudios sobre la vegetación de México. III. Notas sobre la vegetación del Estado de Puebla. Anales del Instituto de Biología. Universidad Nacional Autónoma de México. XIII:417-459.

Miranda F. 1947. Estudios sobre la vegetación de México. V. Rasgos de la vegetación en la cuenca del río Balsas. Revista de la Sociedad Mexicana de Historia Natural 8:95-114.

Miranda F. 1952. La Vegetación de Chiapas. Gobierno del Estado de Chiapas, Tuxtla Gutiérrez.

Miranda F. y Hernández-X. E. 1963. Los tipos de vegetación de México y su clasificación. Boletín de la Sociedad Botánica de México 28:29-179.

Mueller-Dombois D. y Ellenberg H. 1974. Aims and Methods of Vegetation Ecology. John Wiley and Sons, Nueva York.

Murphy P.G. y Lugo A.E. 1995. Dry forest of Central American and the Caribbean. En: Bullock S.H., Mooney H.A. y Medina E. Eds. Seasonally Dry Tropical Forests, pp. 9-34, Cambridge University Press, Cambridge.

Palacio-Prieto J.L., Bocco G., Velázquez A., Mas J.-F., TakakiTakaki F., Victoria A., Luna-González L., Gómez-Rodríguez G., López-García J., Palma M., Trejo-Vázquez I., Peralta A., Prado-Molina J., Rodríguez-Aguilar A., Mayorga-Saucedo R. y González-Medrano F. 2000. La condición actual de los recursos forestales en México: resultados del Inventario Forestal Nacional 2000. Investigaciones Geográficas, Boletín del Instituto de Geografía UNAM 43:183-203.

Pérez-García E.A. 2002. Enclaves de vegetación xerofítica en regiones mésicas: caracterización, análisis de su diversidad florística e importancia en el mantenimiento de floras xerofíticas. Tesis de Maestría (Ecología Básica), Instituto de Ecología, Universidad Nacional Autónoma de México, México, D.F., 154 pp.

Pérez-García E.A. y Meave J.A. 2004. Heterogeneity of xerophytic vegetation of limestone outcrops in a tropical deciduous forest region in southern México. Plant Ecology 175:147-163.

Pérez-García E.A., Meave J. y Gallardo C. 2001. Vegetación y flora de la región de Nizanda, Istmo de Tehuantepec, Oaxaca, México. Acta Botanica Mexicana 56:19-88. 
Pérez-García E.A., Meave J.A. y Gallardo-Cruz J.A. En prensa. Diversidad $\beta$ y diferenciación florística en un paisaje complejo del trópico estacionalmente seco del sur de México. En: Halffter G., Soberón J., Melic Blas A. y Koleff P. Eds. Conversaciones de Diversidad Biológica: el Significado de Alfa, Beta y Gamma. Sociedad Entomológica Aragonesa y Comisión Nacional para el Conocimiento y Uso de la Biodiversidad.

Rico-Gray V., García-Franco J.G., Puch A. y Simá P. 1988. Composition and structure of a tropical dry forest in Yucatan, Mexico. International Journal of Ecology and Environmental Sciences 14:21-29.

Robert A. y Moravie M.-A. 2003. Topographic variation and stand heterogeneity in a wet evergreen forest of India. Journal of Tropical Ecology 19:697-707.

Rzedowski J. 1962. Contribuciones a la fitogeografía florística e histórica de México I. Algunas consideraciones acerca del elemento endémico en la flora mexicana. Boletín de la Sociedad Botánica de México 27:52-65.

Rzedowski J. 1978. Vegetación de México. Editorial Limusa, México, D.F.

Rzedowski J., Medina R. y Calderón de Rzedowski G. 2004. Las especies de Bursera (Burseraceae) en la cuenca superior del río Papaloapan (México). Acta Botanica Mexicana 66:23-151.

Rzedowski J., Medina R. y Calderón de Rzedowski G. 2005. Inventario del conocimiento taxonómico, así como de la diversidad y del endemismo regionales de las especies mexicanas de Bursera (Burseraceae). Acta Botanica Mexicana 70:85-111.

Salas-Morales S.H. 2002. Relaciones entre la heterogeneidad ambiental y la variabilidad estructural de las selvas tropicales secas de la costa de Oaxaca, México. Tesis de Maestría (Biología Ambiental), Facultad de Ciencias, Universidad Nacional Autónoma de México, México, D.F., 101 pp.
Torres-Colín R. 2004. Tipos de vegetación. En: García-Mendoza A.J., Ordóñez M.J. y Briones-Salas M., Eds. Biodiversidad de Oaxaca. pp. 105-117, Instituto de Biología, UNAM-Fondo Oaxaqueño para la Conservación de la Naturaleza-World Wildlife Fund, México, D.F.

Trejo I. 1998. Distribución y diversidad de selvas bajas de México: relaciones con el clima y el suelo. Tesis de Doctorado. Facultad de Ciencias, Universidad Nacional Autónoma de México, México, D.F., 210 pp.

Trejo I. y Dirzo R. 2000. Deforestation of a seasonally dry tropical forest: a national and local analysis in Mexico. Biological Conservation 94:133-142.

Trejo I. y Dirzo R. 2002. Floristic diversity of Mexican seasonally dry tropical forests. Biodiversity and Conservation 11:20632084.

Valencia R.R. 1995. Composition and structure of an Andean forest fragment in eastern Ecuador. En: Churchill S.P., Balslev H., Forero M. y Lutein J.L. Eds. Biodiversity and Conservation of Neotropical Forests, pp. 239-249, The New York Botanical Garden Press, Nueva York.

Valencia R. y Jørgensen P.M. 1992. Composition and structure of a humid montane forest and the Pasochoa Volcano, Ecuador. Nordic Journal of Botany 12:239-247.

Valle-Doménech J.A. 2000. Análisis estructural de una hectárea de selva alta perennifolia en el Monumento Natural Yaxchilán (Chiapas) México. Tesis de Licenciatura. Facultad de Ciencias, Universidad Nacional Autónoma de México, México, D.F., 99 pp.

White D.A. y Hood C.S. 2004. Vegetation patterns and environmental gradients in tropical dry forests of northern Yucatan Peninsula. Journal of Vegetation Science 15:151-160

Fecha de recepción: 3 de febrero de 2005

Versión corregida: 31 de marzo de 2005

Aceptado: 14 de abril de 2005 
Apéndice 1. Valores estructurales y valor de importancia relativa (VIR) para las 145 especies del estrato alto registradas en 30 cuadros de muestreo en el Cerro Verde, Nizanda (Oaxaca), México. $\mathrm{D}=$ densidad; F = frecuencia; $\mathrm{AB}=$ área basal.

\begin{tabular}{|c|c|c|c|c|}
\hline Especie & $\mathrm{D}$ & $\mathrm{F}$ & $\mathrm{AB}\left(\mathrm{m}^{2}\right)$ & VIR \\
\hline Bursera spp. [B. simaruba (L.) Sarg. + B. aff. cinerea Engl.] & 93 & 25 & 182.75 & 22.01 \\
\hline Beaucarnea stricta Lem. & 2 & 2 & 266.76 & 17.31 \\
\hline Euphorbia schlechtendalii Boiss. & 153 & 24 & 22.20 & 15.81 \\
\hline Jacaratia mexicana A.DC. & 17 & 12 & 166.71 & 13.67 \\
\hline Pilosocereus collinsii (Britton et Rose) Byles et G.D.Rowley & 51 & 19 & 60.98 & 10.47 \\
\hline Plumeria rubra L. f. acutifolia (Poir.) Woodson & 40 & 17 & 36.89 & 7.86 \\
\hline Cnidoscolus megacanthus Breckon & 53 & 15 & 14.74 & 7.02 \\
\hline Lysiloma divaricatum (Jacq.) J.F.Macbr. & 24 & 13 & 46.00 & 6.69 \\
\hline Capparis verrucosa Jacq. & 38 & 18 & 16.36 & 6.60 \\
\hline Pseudobombax ellipticum (Kunth) Dugand & 8 & 6 & 76.82 & 6.39 \\
\hline Pachycereus pecten-aboriginum (A.Berger) Britton et Rose & 18 & 6 & 63.96 & 6.26 \\
\hline Pilocarpus racemosus Vahl var. racemosus & 67 & 6 & 6.12 & 5.95 \\
\hline Malpighia emarginata Sessé et Moc. ex D.C. & 32 & 12 & 27.84 & 5.92 \\
\hline Acacia picachensis Brandegee & 10 & 6 & 59.88 & 5.45 \\
\hline Acalypha sp. 09 & 41 & 14 & 1.47 & 5.20 \\
\hline Lonchocarpus longipedicellatus Pittier & 25 & 13 & 14.00 & 4.73 \\
\hline Apoplanesia paniculata C.Presl & 15 & 11 & 29.59 & 4.71 \\
\hline Malpighia mexicana A.Juss. & 24 & 9 & 24.57 & 4.67 \\
\hline Lagrezia monosperma (Rose) Standl. & 34 & 12 & 3.32 & 4.51 \\
\hline Gyrocarpus mocinnoi Espejo & 20 & 11 & 17.00 & 4.25 \\
\hline Hippomane mancinella L. & 19 & 9 & 19.63 & 4.02 \\
\hline Rhamnaceae sp. 02 & 21 & 7 & 22.03 & 3.98 \\
\hline Recchia connaroides (Loes. et Soler) Standl. & 20 & 8 & 16.47 & 3.72 \\
\hline Casearia nitida (L.) Jacq. & 21 & 9 & 10.18 & 3.56 \\
\hline Coursetia sp. 03 & 19 & 11 & 7.00 & 3.55 \\
\hline Sideroxylon stenospermum (Standl.) T.D.Penn. & 6 & 6 & 33.24 & 3.50 \\
\hline Erythrina lanata Rose & 9 & 6 & 25.63 & 3.22 \\
\hline Spondias purpurea L. & 6 & 5 & 30.73 & 3.17 \\
\hline Cedrela salvadorensis Standl. & 7 & 6 & 22.23 & 2.87 \\
\hline Havardia campylacantha (L.Rico et M.Sousa) Barneby et J.W.Grimes & 15 & 5 & 14.72 & 2.78 \\
\hline Aphelandra scabra (Vahl) Sm. & 21 & 8 & 0.37 & 2.78 \\
\hline Bunchosia strigosa Schltdl. & 14 & 8 & 7.82 & 2.77 \\
\hline Aeschynomene compacta Rose & 20 & 8 & 0.94 & 2.74 \\
\hline Bauhinia cookii Rose & 20 & 2 & 15.43 & 2.67 \\
\hline Croton pseudoniveus Lundell & 14 & 6 & 10.41 & 2.60 \\
\hline Neobuxbaumia scoparia (Poselg.) Backeb. & 5 & 3 & 26.82 & 2.53 \\
\hline Desconocida sp. 77 & 11 & 7 & 8.22 & 2.42 \\
\hline Acalypha diversifolia Jacq. var. diversifolia & 20 & 6 & 0.70 & 2.40 \\
\hline Thouinia villosa DC. & 11 & 6 & 10.10 & 2.38 \\
\hline Esenbeckia collina Brandegee & 17 & 5 & 2.21 & 2.12 \\
\hline Morisonia americana L. & 10 & 5 & 9.07 & 2.08 \\
\hline Capparis incana Kunth & 11 & 6 & 3.26 & 1.95 \\
\hline Acalypha sp. 08 & 13 & 6 & 0.88 & 1.93 \\
\hline Buxus bartlettii Standl. & 19 & 2 & 4.43 & 1.91 \\
\hline Sapranthus microcarpus (Donn.Sm.) R.E.Fr. & 8 & 6 & 5.39 & 1.88 \\
\hline Lonchocarpus emarginatus Pittier & 8 & 5 & 4.16 & 1.63 \\
\hline Croton niveus Jacq. & 15 & 3 & 1.04 & 1.58 \\
\hline Nyctaginaceae sp. 07 & 3 & 3 & 13.84 & 1.57 \\
\hline Guaiacum coulteri A.Gray & 4 & 4 & 9.55 & 1.54 \\
\hline Krugiodendron ferreum (Vahl) Urb. & 8 & 4 & 4.80 & 1.51 \\
\hline Thevetia plumeriifolia Benth. & 6 & 5 & 4.12 & 1.49 \\
\hline Stemmadenia obovata (Hook. et Arn.) K.Schum. & 7 & 5 & 1.56 & 1.40 \\
\hline Yucca sp. nov. ined. & 11 & 2 & 4.73 & 1.38 \\
\hline
\end{tabular}


Estructura y diversidad de la SElva baja CADUCifolia del Cerro Verde, OAXacA

Apéndice 1. Continuación

\begin{tabular}{|c|c|c|c|c|}
\hline Especie & $\mathrm{D}$ & $\mathrm{F}$ & $\mathrm{AB}\left(\mathrm{m}^{2}\right)$ & VIR \\
\hline Jacquinia macrocarpa Cav. & 6 & 5 & 1.52 & 1.33 \\
\hline Senna holwayana (Rose) H.S.Irwin et Barneby & 9 & 4 & 0.85 & 1.33 \\
\hline Lonchocarpus sp. 05 & 8 & 4 & 1.25 & 1.28 \\
\hline Bahuinia seleriana Harms & 11 & 2 & 2.46 & 1.24 \\
\hline Lonchocarpus sp. 03 & 7 & 4 & 1.38 & 1.22 \\
\hline Leguminosae sp. 14 & 5 & 5 & 0.88 & 1.22 \\
\hline Lonchocarpus torresiorum M.Sousa & 6 & 4 & 1.99 & 1.19 \\
\hline Senna atomaria (L.) H.S.Irwin et Barneby & 3 & 3 & 7.50 & 1.17 \\
\hline Manihot oaxacana D.J.Rogers et Appan & 5 & 4 & 1.39 & 1.09 \\
\hline Asteraceae sp. 02 & 6 & 4 & 0.25 & 1.08 \\
\hline Capparis indica (L.) Druce & 6 & 3 & 2.25 & 1.05 \\
\hline Celasteraceae sp. 03 & 7 & 3 & 1.02 & 1.04 \\
\hline Croton fragilis Kunth & 7 & 3 & 0.71 & 1.02 \\
\hline Hibiscus kochii Fryxell & 5 & 4 & 0.07 & 1.01 \\
\hline Grajalesia fasciculata (Standl.) Miranda & 1 & 1 & 11.95 & 0.99 \\
\hline Sinclairia andrieuxii (DC.) H.Rob. et Brettel & 4 & 4 & 0.41 & 0.96 \\
\hline \multicolumn{5}{|l|}{ Sideroxylon obtusifulium (Roem. et Schult.) T.D.Penn. } \\
\hline subsp. buxifolium (Roem. et Schult.) T.D.Penn. & 2 & 2 & 7.65 & 0.95 \\
\hline Pisonia aculeata L. & 8 & 1 & 3.42 & 0.93 \\
\hline Erythroxylum havanense Jacq. & 5 & 3 & 0.55 & 0.87 \\
\hline Roldana eriophylla (Greenm.) H.Rob. et Brettel & 4 & 3 & 0.18 & 0.78 \\
\hline Physalis arborescens L. & 4 & 3 & 0.10 & 0.77 \\
\hline Malmea depressa (Baill.) R.E.Fr. & 1 & 1 & 8.04 & 0.74 \\
\hline Xanthoxylum fagara (L.) Sarg. & 3 & 3 & 0.46 & 0.73 \\
\hline Desconocida sp. 78 & 1 & 1 & 7.64 & 0.72 \\
\hline Bignoniaceae sp. 06 & 7 & 1 & 1.11 & 0.71 \\
\hline Nyctaginaceae sp. 08 & 3 & 2 & 2.82 & 0.71 \\
\hline Ficus cotinifolia Kunth & 3 & 3 & 0.14 & 0.71 \\
\hline Heteropterys laurifolia (L.) A.Juss. & 4 & 2 & 1.64 & 0.71 \\
\hline Bakeridesia integerrima (Hook.f.) D.M.Bates & 3 & 3 & 0.11 & 0.71 \\
\hline Apocynaceae sp. 02 & 5 & 2 & 0.38 & 0.69 \\
\hline Jatropha alamanii Müll.Arg. & 2 & 2 & 3.24 & 0.67 \\
\hline Arrabidaea costaricensis (Kränzl.) A.H.Gentry & 4 & 2 & 0.91 & 0.66 \\
\hline Amyris aff. sylvatica Jacq. & 4 & 2 & 0.62 & 0.64 \\
\hline Lonchocarpus lanceolatus Benth. & 6 & 1 & 0.93 & 0.63 \\
\hline Asteraceae sp. 01 & 4 & 2 & 0.44 & 0.63 \\
\hline Myrtaceae sp. 03 & 4 & 2 & 0.15 & 0.61 \\
\hline Randia thurberi S.Watson & 4 & 2 & 0.10 & 0.61 \\
\hline Triumfetta sp. 02 & 3 & 2 & 1.11 & 0.60 \\
\hline Stemmadenia eubracteata Woodson & 3 & 2 & 0.27 & 0.55 \\
\hline Zapoteca portoricensis (Jacq.) H.M.Hern. & 3 & 2 & 0.25 & 0.55 \\
\hline Rutaceae sp. 02 & 4 & 1 & 1.70 & 0.55 \\
\hline Eugenia aff. salamensis Donn.Sm. & 2 & 2 & 1.25 & 0.55 \\
\hline Cordia inermis (Mill.) I.M.Johnst. & 3 & 2 & 0.17 & 0.54 \\
\hline Marsdenia coulteri Hemsl. & 3 & 2 & 0.11 & 0.54 \\
\hline Lasianthaea fruticosa (L.) K.M.Becker var. fruticosa & 3 & 2 & 0.04 & 0.54 \\
\hline Asteraceae sp. 21 & 5 & 1 & 0.45 & 0.54 \\
\hline Annona squamosa L. & 2 & 2 & 0.89 & 0.52 \\
\hline Urticaceae sp. 02 & 2 & 2 & 0.54 & 0.50 \\
\hline Arrabidaea floribunda (Kunth) Loes. & 2 & 2 & 0.34 & 0.49 \\
\hline Luehea sp. 01 & 2 & 2 & 0.16 & 0.48 \\
\hline \multicolumn{5}{|l|}{ Pittocaulon velatum (Greenm.) H.Rob. et Brettel var. tzimolense } \\
\hline (T.M.Barkley) B.L.Clark & 2 & 2 & 0.13 & 0.47 \\
\hline Capsicum annuum L. var. grabriusculum (Dunal) Heiser et Pickersgill & 2 & 2 & 0.08 & 0.47 \\
\hline
\end{tabular}


José A. Gallardo-Cruz, Jorge A. Meave y Eduardo A. Pérez-García

Apéndice 1. Continuación

\begin{tabular}{|c|c|c|c|c|}
\hline Especie & $\mathrm{D}$ & $\mathrm{F}$ & $\mathrm{AB}\left(\mathrm{m}^{2}\right)$ & VIR \\
\hline Malvaceae sp. 01 & 2 & 2 & 0.01 & 0.47 \\
\hline Justicia caudata A.Gray & 4 & 1 & 0.41 & 0.46 \\
\hline Desconocida sp. 67 & 1 & 1 & 2.81 & 0.41 \\
\hline Randia cinerea (Fernald) Standl. & 3 & 1 & 0.36 & 0.39 \\
\hline Bucida macrostachya Standl. & 3 & 1 & 0.23 & 0.38 \\
\hline Capparis lundellii Standl. & 3 & 1 & 0.14 & 0.38 \\
\hline Pedilanthus calcaratus Schltdl. & 3 & 1 & 0.14 & 0.38 \\
\hline $\begin{array}{l}\text { Jacobinia spp. [J. candicans (Nees) Benth. } \\
\text { et Hook.f. + J. mollis Greenm.] }\end{array}$ & 3 & 1 & 0.13 & 0.38 \\
\hline Coccoloba liebmannii Lindau & 2 & 1 & 1.17 & 0.38 \\
\hline Castela retusa Liebm. & 2 & 1 & 0.53 & 0.33 \\
\hline Machaerium pittieri Macbr. & 2 & 1 & 0.33 & 0.32 \\
\hline Tabebuia impetiginosa (Mart. ex DC.) Standl. & 1 & 1 & 1.31 & 0.32 \\
\hline Karwinskia humboldtiana (Roem. et Schult.) Zucc. & 2 & 1 & 0.09 & 0.31 \\
\hline Asteraceae sp. 24 & 2 & 1 & 0.09 & 0.31 \\
\hline Randia obcordata S.Watson & 2 & 1 & 0.05 & 0.30 \\
\hline Acalypha sp. 06 & 2 & 1 & 0.03 & 0.30 \\
\hline Asteraceae sp. 26 & 2 & 1 & 0.03 & 0.30 \\
\hline Piptadenia flava (Spreng. ex DC.) Benth. & 1 & 1 & 0.27 & 0.25 \\
\hline Caesalpinia platyloba S.Watson & 1 & 1 & 0.26 & 0.25 \\
\hline Myrtaceae sp. 05 & 1 & 1 & 0.23 & 0.25 \\
\hline Acacia tenuifolia (L.) Willd. & 1 & 1 & 0.16 & 0.24 \\
\hline Ipomoea sp. 03 & 1 & 1 & 0.11 & 0.24 \\
\hline Heteropterys cotinifolia A.Juss. & 1 & 1 & 0.10 & 0.24 \\
\hline Asteraceae sp. 10 & 1 & 1 & 0.08 & 0.24 \\
\hline Asteraceae sp. 12 & 1 & 1 & 0.07 & 0.24 \\
\hline Achatocarpus nigricans Triana & 1 & 1 & 0.07 & 0.24 \\
\hline Amphipterygium adstringens (Schltdl.) Standl. & 1 & 1 & 0.07 & 0.24 \\
\hline Tiliaceae sp. 02 & 1 & 1 & 0.06 & 0.24 \\
\hline Verbesina crocata (Cav.) Less. & 1 & 1 & 0.06 & 0.24 \\
\hline Serjania triquetra Radlk. & 1 & 1 & 0.04 & 0.24 \\
\hline Forchhammeria pallida Liebm. & 1 & 1 & 0.03 & 0.23 \\
\hline Solanum glaucescens Zucc. & 1 & 1 & 0.03 & 0.23 \\
\hline Sapindaceae sp. 04 & 1 & 1 & 0.01 & 0.23 \\
\hline Iresine calea (Ibáñez) Standl. & 1 & 1 & 0.01 & 0.23 \\
\hline Hamelia patens Jacq. & 1 & 1 & 0.01 & 0.23 \\
\hline Chromolaena collina (DC.) R.M.King et H.Rob. & 1 & 1 & 0.01 & 0.23 \\
\hline Sapium appendiculatum (Müll.Arg.) Pax et K.Hoffm. & 1 & 1 & 0.01 & 0.23 \\
\hline Mimosa goldmanii B.L.Rob. & 1 & 1 & 0.01 & 0.23 \\
\hline Asteraceae sp. 25 & 1 & 1 & 0.01 & 0.23 \\
\hline Pedilanthus tithymaloides (L.) Poit. subsp. tithymaloides & 1 & 1 & 0.01 & 0.23 \\
\hline
\end{tabular}


Apéndice 2. Listado florístico de las 49 especies que sólo fueron registradas para el estrato bajo en 150 subcuadros $\left(4 \mathrm{~m}^{2}\right)$ de muestreo de selva baja caducifolia en el Cerro Verde, Nizanda, Oaxaca.

\begin{tabular}{|c|c|}
\hline Familias y especies & Familias y especies \\
\hline Acanthaceae & Leguminosae \\
\hline Carlowrightia arizonica A.Gray & Leguminosae sp. 17 \\
\hline Siphonoglossa sessilis (Jacq.) D.N.Gibson & Senna fruticosa (Mill.) H.S.Irwin et Barneby \\
\hline Agavaceae & Senna pallida (Vahl) H.S.Irwin et Barneby \\
\hline Agave angustifolia Haw. & Malpighiaceae \\
\hline Amaranthaceae & Hiraea reclinata Jacq. \\
\hline Alternanthera mexicana Moq. & Malvaceae \\
\hline Amaranthaceae sp. 01 & Malvaceae sp. 06 \\
\hline Iresine aff. interrupta Benth. & Malvaceae sp. 13 \\
\hline Anacardiaceae & Phytolacaceae \\
\hline Astronium graveolens Jacq. & Petiveria alliacea L. \\
\hline Apocynaceae & Poaceae \\
\hline Haplophyton cimicidum A.DC. & Lasiacis ruscifolia (Kunth) Hitchc. \\
\hline Mesechites trifida (Jacq.) Müll.Arg. & Polygonaceae \\
\hline Asclepiadaceae & Coccoloba barbadensis Jacq. \\
\hline Asclepiadaceae sp. 08 & Portulacaceae \\
\hline Araceae & Portulaca oleracea L. \\
\hline Anthurium nizandense Matuda & Talinum triangulare (Jacq.) Willd. \\
\hline Philodendron hederaceum (Jacq.) Schott & Rubiaceae \\
\hline Asteraceae & Randia sp. 06 \\
\hline Asteraceae sp. 13 & Rubiaceae sp. 04 \\
\hline Asteraceae sp. 22 & Rubiaceae sp 13 \\
\hline Asteraceae sp. 23 & Sapindaceae \\
\hline Bidens squarrosa Kunth & Sapindaceae sp. 07 \\
\hline Chromolaena odorata (L.) R.M.King et H.Rob. & Solanaceae \\
\hline Perymenium aff. grande Hemsl. & Lycianthes lenta (Cav.) Bitter \\
\hline Bignoniaceae & Nicotiana plumbaginifolia Viv. \\
\hline Adenocalymma inundatum C.Mart. ex DC. & Solanum americanum Mill. \\
\hline Bignoniaceae sp. 14 & Sterculiaceae \\
\hline Bromeliaceae & Ayenia glabra S.Watson \\
\hline Bromelia palmeri Mez & Tiliaceae \\
\hline Hechtia rosea E.Morren ex Baker & Heliocarpus sp. 01 \\
\hline Cactaceae & Tiliaceae sp. 02 \\
\hline Acanthocereus tetragonus (L.) Hummelinck & Urticaceae \\
\hline Commelinaceae & Urticaceae sp. 03 \\
\hline \multirow{2}{*}{\multicolumn{2}{|c|}{$\begin{array}{l}\text { Commelina rufipes Seub. var. glabrata (D.R.Hunt.) } \\
\text { Faden et D.R.Hunt }\end{array}$}} \\
\hline & \\
\hline \multicolumn{2}{|l|}{ Euphorbiaceae } \\
\hline \multicolumn{2}{|l|}{ Acalypha sp. 05} \\
\hline \multicolumn{2}{|l|}{ Acalypha sp. 07} \\
\hline Euphorbia segoviensis (Klotzsch et Garcke) Boiss. & \\
\hline
\end{tabular}

